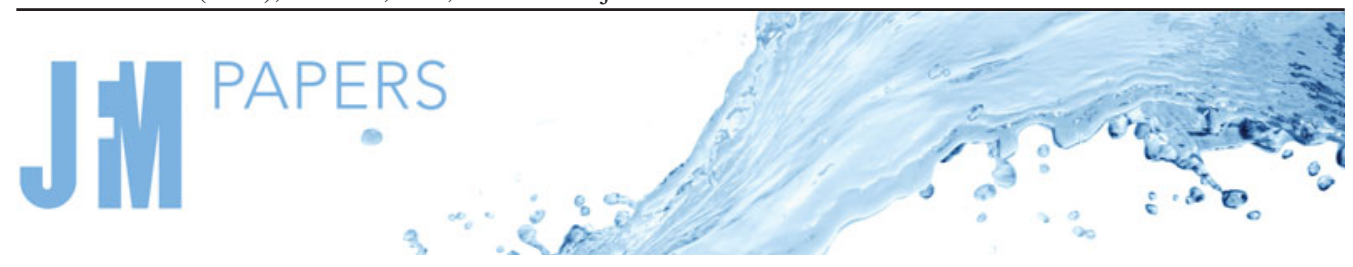

\title{
Direct numerical simulation of a turbulent boundary layer over a bump with strong pressure gradients
}

\author{
Riccardo Balin ${ }^{1} \uparrow^{\dagger}$ and K.E. Jansen ${ }^{1}$ \\ ${ }^{1}$ Ann and H.J. Smead Aerospace Engineering Sciences, University of Colorado Boulder, Boulder, \\ CO 80309, USA
}

(Received 17 October 2020; revised 11 February 2021; accepted 5 April 2021)

The turbulent boundary layer over a Gaussian-shaped bump is computed by direct numerical simulation of the incompressible Navier-Stokes equations. The two-dimensional bump causes a series of strong pressure gradients alternating in rapid succession. At the inflow, the momentum thickness Reynolds number is approximately 1000 and the boundary layer thickness is $1 / 8$ of the bump height. Direct numerical simulation results show that the strong favourable pressure gradient (FPG) causes the boundary layer to enter a relaminarization process. The near-wall turbulence is significantly weakened and becomes intermittent, however, relaminarization does not complete. The streamwise velocity profiles deviate above the standard logarithmic law and the Reynolds shear stress is reduced. The strong acceleration also suppresses the wall-shear normalized turbulent kinetic energy production rate. At the bump peak, where the FPG switches to an adverse gradient (APG), the near-wall turbulence is suddenly enhanced through a partial retransition process. This results in a new highly energized internal layer which is more resilient to the strong APG and only produces incipient flow separation on the downstream side. In the strong FPG and APG regions, the inner and outer layers become largely independent of each other. The near-wall region responds to the pressure gradients and determines the skin friction. The outer layer behaves similarly to a free shear layer subject to pressure gradients and mean streamline curvature effects. Results from a RANS simulation of the bump are also discussed and clearly show the lack of predictive capacity of the near-wall pressure gradient effects on the mean flow.

Key words: turbulent boundary layers, turbulence simulation

$\dagger$ Email address for correspondence: riccardo.balin@ colorado.edu

(C) The Author(s), 2021. Published by Cambridge University Press. This is an Open Access article, distributed under the terms of the Creative Commons Attribution licence (http://creativecommons.org/ licenses/by/4.0/), which permits unrestricted re-use, distribution, and reproduction in any medium, provided the original work is properly cited. 


\section{R. Balin and K.E. Jansen}

\section{Introduction}

Turbulent boundary layers undergoing pressure gradients and separation have been the subject of a large number of studies due to their ubiquity in science and engineering. Indeed, a deep understanding of their complex physics is necessary for the accurate prediction and design of many engineering systems. While still limited to low Reynolds numbers and small domains, the continuous improvement in computational resources has made investigation by direct numerical simulation (DNS) increasingly popular. Direct numerical simulation has not only been of great complement to experimental work in the study of boundary layer physics, but has also provided detailed and dense high-fidelity data for the evaluation and improvement of all lower-fidelity turbulence models, from large eddy simulation (LES) to Reynolds averaged Navier-Stokes (RANS) closures (Abe et al. 2012; Coleman, Rumsey \& Spalart 2018; Matai \& Durbin 2019; Balin, Jansen \& Spalart 2020).

A significant number of DNS of boundary layers with pressure gradients and separation have focused on the flow over a flat plate. In these studies, the boundary layer is subjected first to an adverse pressure gradient (APG) which causes the flow to separate, followed a favourable pressure gradient (FPG) in order to reattach the flow. Pioneering work in this area was performed by Spalart \& Watmuff (1993), although in their case the boundary layer did not separate. This was followed by a great number of studies, including Spalart \& Coleman (1997), Na \& Moin (1998), Skote \& Henningson (2002), Manhart \& Friedrich (2002), Abe et al. (2012), Abe (2017), Kitsios et al. (2016), Coleman et al. (2018), performing simulations of the same kind at increasingly higher values of the momentum thickness Reynolds number $\operatorname{Re}_{\theta}$.

Flat plate boundary layer flows provide valuable insight into smooth-body shallow separation caused by continued APG effects, which is a well known deficiency of turbulence models. However, they lack two important characteristics that are often present in engineering applications, namely strong FPGs, in particular a strong FPG upstream of the APG, and strong streamline curvature. The effects of these two phenomena on boundary layer turbulence were initially investigated experimentally with flows over bumps and hills (Tsuji \& Morikawa 1976; Baskaran, Smits \& Joubert 1987, 1991; Webster, DeGraaff \& Eaton 1996). In all cases, the breakdown of the logarithmic law with the streamwise velocity deviating above it was observed in the strong FPG region. The presence of internal layers was also reported in these flows, with Baskaran et al. (1991) noting a decoupling of the inner and outer layers and the latter behaving as a free shear layer. Curvature effects were most pronounced in the outer layer, while changes due to the pressure gradient initially appeared in the inner region.

The experimental bump flow of Webster et al. (1996) was simulated with wall-resolved LES by Wu \& Squires (1998), Cavar \& Meyer (2011) and Matai \& Durbin (2019), confirming the significant departure above the logarithmic law in the FPG region and the presence of internal layers. Matai \& Durbin (2019) expanded the study by considering a family of bumps of increasing height. For all cases, they observed a plateau or a rapid oscillation in the skin friction at the start of the APG. They also showed that the non-dimensional FPG

$$
\Delta_{p}=\frac{v}{\rho u_{\tau}^{3}} \frac{\partial p}{\partial s},
$$

exceeded the value of -0.018 identified by Patel (1965) marking the start of a relaminarization process. Note that in (1.1), $v$ is the kinematic viscosity, $\rho$ is the fluid density, $p$ is the mean static pressure at the wall, $u_{\tau}$ is the friction velocity and $s$ is 


\section{DNS of a boundary layer with strong pressure gradients}

the streamwise direction. Uzun \& Malik (2018) performed wall-resolved LES of the NASA wall-mounted hump and compared the simulation results with the experiments of Greenblatt et al. (2006). Both numerical and experimental data showed the presence of a plateau in the skin friction coefficient profile where the FPG was strong. It was noted that in this region the boundary layer was undergoing a relaminarization process according to the acceleration parameter based on the edge velocity $U_{e}$,

$$
K=\frac{v}{U_{e}^{2}} \frac{\partial U_{e}}{\partial s},
$$

but full relaminarization was not achieved.

Although often not the focus of the aforementioned studies on bump and hill flows, all reported a region of strong FPG with large deviations from standard turbulent boundary layer behaviour. Some of them even mentioned signs of relaminarization (also known as reverse transition). Patel (1965) noted the deviation of the streamwise velocity profile above the logarithmic law for large enough negative values of $\Delta_{p}$. They supposed the breakdown of the law was due to the process of reversion to laminar flow and proposed a tentative critical value of $\Delta_{p}=-0.018$. However, they noted that $\Delta_{p}$ did not describe the near-wall flow completely and in a later study proposed the use of a different quantity (Patel \& Head 1968). They argued that the non-dimensional shear stress gradient

$$
\Delta_{\tau}=\frac{\nu \alpha}{\rho u_{\tau}^{3}},
$$

is more universal and identified a critical value of -0.009 for the departure above the logarithmic law and relaminarization. Note that in (1.3), $\alpha$ is the gradient of the total shear stress in the wall-normal direction, $n$, across the viscous sublayer such that $\tau=$ $\tau_{w}+\alpha n$. Bradshaw (1969) later corrected the critical value for $\Delta_{\tau}$ to -0.013 and noted that it marks the beginning of the logarithmic law overshoot and not necessarily the start of a relaminarization process. Narasimha \& Sreenivasan $(1973,1979)$ also noted that the departure from the logarithmic law occurs upstream of the reverse transitional process. They proposed critical values of $\Delta_{p}=-0.025$ and $K=3 \times 10^{-6}$ as further indication of the onset of relaminarization. These critical values on the pressure gradient were supported by a DNS of sink flows (Spalart 1986).

Despite APG effects and separation receiving most of the attention in the literature, it will become apparent throughout this paper that a complete understanding and accurate modelling of FPG effects are critical, yet currently lacking (Uzun \& Malik 2018; Matai \& Durbin 2019; Balin et al. 2020). Additionally, a relatively limited number of studies, especially numerical ones, discuss the details of turbulence undergoing strong acceleration, relaminarization and retransition (the process describing the return to turbulence after relaminarization) over complex geometries. There is, in fact, considerable uncertainty as to whether the critical values of the pressure gradient parameters ((1.1)-(1.3)) are valid beyond flat plate flows. The need for additional investigations into strong FPG flows, therefore, certainly exists and DNS are especially well-suited for this purpose since so much of the FPG effects are located near the wall. The work presented in this paper addresses this need by offering a novel case of a boundary layer undergoing strong pressure gradients and streamline curvature effects of alternating sign with the purpose of increasing the understanding of these types of flows and provide detailed data for the improvement of lower fidelity turbulence models.

A DNS of the turbulent boundary layer flowing over a Gaussian bump is performed. The flow is accelerated on the upstream side by a strong FPG, and then is quickly 


\section{R. Balin and K.E. Jansen}

decelerated on the downstream side by an APG leading to incipient separation. At the relatively low Reynolds number chosen (Re based on the momentum thickness is approximately 1000 at the inflow), the FPG acting over an extended length $(\approx 20$ boundary layer thicknesses) causes the onset of relaminarization and a significant weakening of the near-wall turbulence. The boundary layer, however, does not relaminarize completely and stays intermittently turbulent. At the peak of the bump, the weakened near-wall turbulence experiences a sudden enhancement in intensity due to partial retransition, which in turn leads to an atypical skin friction response and a more resilient boundary layer. The DNS was designed to focus on the part of the flow leading up to incipient separation rather than downstream of it, with additional emphasis on the FPG region. The discussion of results reflects this choice, therefore incipient separation and the redevelopment of the boundary layer are not discussed in this paper.

The present DNS is part of a larger joint study with other research groups and two recent conference publications on DNS of this flow already exist (Balin et al. 2020; Uzun \& Malik 2020). The former presented preliminary results from this work to evaluate wall-modelled LES in the FPG and retransition regions, while the latter takes a more holistic approach with a greater emphasis on the incipient separation and subsequent recovery, making all three papers highly complementary. While both groups simulate the same $\operatorname{Re}_{L}=1 \times 10^{6}$, key differences are present between this paper and Uzun \& Malik (2020). In particular, this paper primarily focuses on the strong FPG region, discussing the flow physics involved in relaminarization from strongly accelerated flows, and grounding it in the body of literature. This clearly distinguishes it from the conference papers which only touched upon these topics.

This paper is organized as follows. Section 2 describes in detail the flow problem chosen and the numerical approach taken to obtain a solution with DNS. Section 3 presents and discusses the results obtained from the DNS. Finally, $§ 4$ offers some concluding remarks.

\section{Numerical set-up}

\subsection{Problem definition}

The flow computed in this study is the turbulent boundary layer over the prismatic extrusion of a two-dimensional (2-D) Gaussian-shaped bump. The surface is defined by the following equation which depends on the height parameter $h$ and the length parameter $x_{0}$, and is shown by the black curve on the lower surface of the domain in figure 1:

$$
y(x)=h \exp \left(-\left(x / x_{0}\right)^{2}\right) .
$$

Note that the $x$ coordinate is aligned with the free stream flow far upstream of the bump, the $y$ coordinate is vertical and normal to the free stream and the $z$ coordinate is aligned with the spanwise direction. Moreover, (2.1) defines the entire lower surface of the domain, meaning that there is no flat-plate region on either side of the bump and the curvature is everywhere continuous. It is important to mention that the geometry selected for this DNS is exactly the centreline of a three-dimensional (3-D) bump developed at The Boeing Company (Slotnick 2019) and studied experimentally at the University of Washington (Williams et al. 2020). Detailed comparisons between the experimental results and the DNS, however, are deferred to future work due the current availability of the wind tunnel data.

To maintain similarities between the two geometries, the 2-D Gaussian bump height and length parameters were matched at $h / L=0.085$ and $x_{0} / L=0.195$, where $L=$ $0.9144 \mathrm{~m}$ is the length of the square cross-section of the wind tunnel used for the $3-\mathrm{D}$ 
DNS of a boundary layer with strong pressure gradients

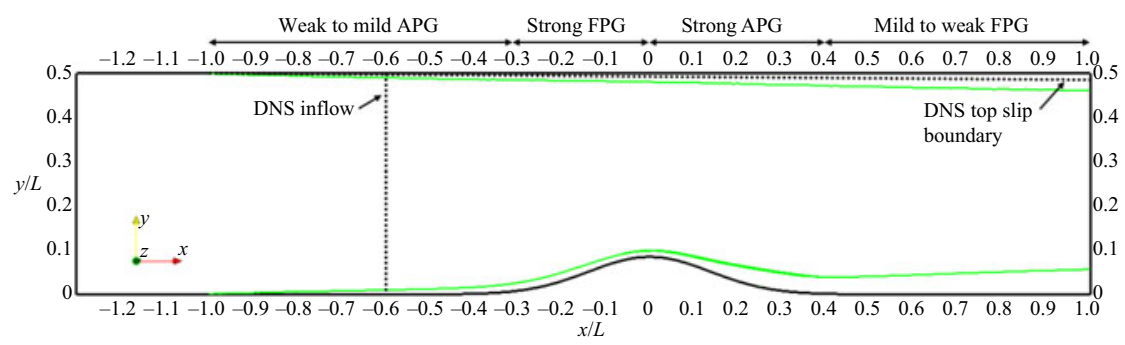

Figure 1. The solid black curves outline the full domain of the bump flow, while the green curves show the boundary layer thickness on both no-slip walls predicted by preliminary RANS. The black dotted lines mark the modified inflow and top boundaries for the DNS.

bump experiments. The flow studied has a Reynolds number of $R e_{L}=1.0 \times 10^{6}$, corresponding to $\operatorname{Re}_{h}=85000$ when measured against the bump height. The free stream velocity is $U_{\infty}=16.40 \mathrm{~m} \mathrm{~s}^{-1}$, which at standard sea level conditions results in a small enough Mach number $\left(M_{\infty}=0.045\right)$ to treat this flow as incompressible. At the location of the inlet to the DNS, shown by the dotted vertical line in figure 1, the momentum thickness Reynolds number is $\operatorname{Re}_{\theta}=1050$ and the boundary layer thickness is approximately $1 / 8$ of the bump height. Due to the boundary layer being thinner than the bump height, the flow is more characteristic of a hill rather than other bumps present in the literature (Webster et al. 1996; Greenblatt et al. 2006), however, the term 'bump' is retained.

Other boundary conditions defining the entire flow domain in figure 1 are as follows. Two solid no-slip walls are present; the bump on the lower surface defined entirely by (2.1) and a flat top surface located at $y / L=0.5$. The origin of the top and bottom boundary layers is at $x / L=-1$, coinciding with the leading edges of the two no-slip walls (Slotnick 2019; Williams et al. 2020). Finally, the top and bottom surfaces are extended upstream of this location and symmetry boundary conditions are prescribed there. This allows a uniform velocity in the $x$ direction equal to $U_{\infty}$ to be imposed at the inflow $(x / L \approx-1.3)$ and the boundary layer origin to be modelled correctly.

\subsection{Solution approach}

While figure 1 describes the entire flow domain of the 2-D Gaussian bump, only a fraction of it could be included in the DNS. The inflow was moved downstream to $x / L=-0.6$ as shown by the dotted line in figure 1. Moreover, the top wall was slanted according to a profile fitted to the displacement thickness of the boundary layer computed with a preliminary 2-D RANS simulation of the entire flow domain (see figure 1). This was done to reproduce the constriction effects of the boundary layer growing on the top wall while keeping the cost of the simulation manageable. The preliminary RANS was carried out using the Spalart-Allmaras (SA) one-equation model (Spalart \& Allmaras 1994) augmented with the rotation and streamline curvature (SARC) correction (Spalart \& Shur 1997; Shur et al. 2000) on the full domain. Additionally, a low-Reynolds-number modification proposed in Coleman et al. (2018) was applied to correct the underprediction of the skin friction coefficient by the SA model at low $R e_{\theta}$. The spanwise width of the DNS domain was set to $4.7 \delta_{995_{i n}}$, where $\delta 995_{i n}$ is the inflow $99.5 \%$ boundary layer thickness, and periodicity was enforced.

Due to the growth of the boundary layer over the bump, as shown in figure 1, it was necessary to determine whether a periodic boundary condition over the spanwise width could introduce some confinement effects in the region focused upon for this study 


\section{R. Balin and K.E. Jansen}

(defined as the portion of the flow leading up to incipient separation $(-0.60 \leq x / L \leq$ 0.19), with particular emphasis on the FPG and the bump peak $(-0.29 \leq x / L \leq 0.10)$, where the boundary layer growth relative to the inflow is only moderate). Confinement effects were studied by performing two wall-modelled LES (known as WMLES) of the same flow described here, with the only difference between them being that the period was increased by a factor of two for one of the simulations. The details of the wall-modelled LES with the DNS domain are discussed in Balin et al. (2020), wherein it was shown that the outer layer Reynolds stresses and boundary layer thickness in the region of the flow focused upon are captured accurately. Since the spanwise period affects the largest scales, which are well captured in both simulations, this exercise is representative of possible confinement effects experienced by the DNS. No significant differences were observed in the flow and turbulent quantities (skin friction, pressure gradient, velocity and Reynolds stresses) between the narrow and wide domain simulations. Consequently, the solution in the region of focus of this DNS is considered to be free of any confinement effects from the spanwise period chosen.

The boundary conditions enforced in the DNS were as follows. The bump surface was treated as a no-slip wall. The top surface was modelled as an inviscid wall offset by the RANS predicted displacement thickness described above with zero transpiration (zero velocity component normal to the surface) and zero traction. At the outflow, weak enforcement of zero pressure was applied along with zero traction. Effects from this boundary condition on the interior domain are contained within a streamwise distance of one local boundary layer thickness and thus did not affect the upstream solution. At the inflow, the synthetic turbulence generator (STG) of Shur et al. (2014) was selected to introduce unsteady flow into the domain, which has been shown to produce realistic turbulence a short distance downstream of the inlet for both wall-modelled LES (Shur et al. 2014) and DNS (Spalart et al. 2017; Wright et al. 2020). The mean Reynolds stress and velocity profiles required by the STG method were extracted from an additional 2-D RANS simulation at the inflow location of $x / L=-0.6$ for which the top surface was modified according to the dotted line in figure 1 and modelled as a slip wall. This was done intentionally to extract profiles along the entire height of the inflow plane consistent with the DNS domain and boundary conditions.

The computational grid used for the DNS of the bump was structured with a total of 554 million points. It possesses the following spacing: $\Delta s^{+} \leq 15, \Delta z^{+}<8, \Delta n_{1}^{+}=0.1$ and $\Delta n_{\max }^{+}<10$, where $(s, n, z)$ is the bump aligned coordinate system ( $s$ and $n$ are tangent and normal to the bump surface, respectively). Moreover, the ( $)^{+}$superscript signifies scaling by wall units using the mean friction velocity $u_{\tau}$ and viscous length scale $l_{v}=v / u_{\tau}$ obtained from the DNS. Note that the streamwise spacing $\Delta s^{+} \leq 15$ was achieved everywhere by using the local value of the friction velocity computed by preliminary RANS in the design of the grid. Additionally, the growth and decay across adjacent elements was limited to $1 \%$ in the streamwise direction, resulting in a smooth variation of $\Delta s$. Close to the separation region predicted by RANS, where $u_{\tau}$ becomes ill-defined, a maximum spacing of $\Delta s / L=4.0 \times 10^{-4}$ (20 plus units based on the inflow $u_{\tau}$ ) was enforced to maintain adequate spacing. The wall spacing $\Delta n_{1}^{+}=0.1$ was set in a similar manner, with a growth factor limited to $5 \%$.

The DNS was initialized from an instantaneous solution of a wall-modelled LES computed on the same domain with the same boundary conditions (Balin et al. 2020). Integration of the flow was carried out for one full domain flow-through time before accumulation of statistics. Past the transient phase, time- and span-averaged statistics were accumulated for a total time $T$ long enough to satisfy $T>100 t_{\text {eddy }}$ everywhere in 


\section{DNS of a boundary layer with strong pressure gradients}

the region of this bump flow the paper focuses on. Note that the eddy-turnover time is defined based on the local edge velocity $\tilde{U}_{e}$ and boundary layer thickness $\tilde{\delta}_{995}$ as $t_{e d d y}=\tilde{U}_{e} / \tilde{\delta}_{995}$ (see (3.2)-(3.5)). The non-dimensional time step size was $\Delta t^{+}=0.11$ based on the maximum average friction velocity of the DNS, which ensured a maximum Courant-Friedrichs-Lewy (known as CFL) number below one at each time step.

All simulations presented in this work were performed with a stabilized finite element method (Whiting \& Jansen 1999) using trilinear hexahedral elements and second-order accurate, fully implicit time integration (Jansen, Whiting \& Hulbert 2000). The accuracy of DNS with this method has been shown for a channel flow in Trofimova, Tejada-Martinez \& Jansen (2009), in which trilinear hexahedral elements were also used. Stabilization and time integration parameters (which affect numerical dissipation) chosen for this DNS follow the work of Trofimova et al. (2009). It is worth pointing out that a DNS of a zero pressure gradient (ZPG) flat plate boundary layer was performed as the initial step in this study. The details and results of this preliminary DNS are described in Wright et al. (2020), wherein numerical and experimental validation data was accurately reproduced through the use of the same STG inflow method and very similar grid resolution to those described above for the Gaussian bump.

\section{Results and discussion}

\subsection{Full bump flow}

Time- and span-averaged pressure and skin friction coefficient profiles on the surface of the entire Gaussian bump obtained from the DNS are presented in figure 2. The coefficients are defined in the following, where $p_{\text {ref }}$ is the reference wall pressure at location $x / L=-0.60$ and $\tau_{w}$ is the wall shear stress rotated to the curvilinear coordinates $(s, n, z)$ :

$$
C_{p}=\frac{p-p_{r e f}}{\frac{1}{2} \rho_{\infty} U_{\infty}^{2}} \quad C_{f}=\frac{\tau_{w}}{\frac{1}{2} \rho_{\infty} U_{\infty}^{2}} .
$$

In this figure, the solution from the preliminary 2-D RANS simulation with the SARC-low-Re closure used to obtain the inflow profiles is also shown for comparison. Additionally, results without the curvature correction (SA-low-Re) are included and discussed later in this section. Finally, $C_{p}$ data over the centreline of the 3-D bump from the experiments of Williams et al. (2020) are compared with the results from a RANS simulation of the same 3-D geometry. Given that the boundary conditions of the 3-D simulation are the same as described in $\S 2.1$ for the 2-D Gaussian bump (i.e. figure 1 accurately depicts the centreline of the 3-D bump), this comparison provides high confidence in the numerical set-up and the relevance of the boundary layer physics studied herein.

Very good agreement between the DNS and SARC-low-Re RANS is obtained for the pressure coefficient, indicating the successful choice of the DNS subdomain and boundary conditions. The $C_{p}$ profile also outlines the series of pressure gradients experienced by the boundary layer. In this subsection, discussion is confined to overall flow but, in doing so, three regions classified by pressure gradient will be identified for more detailed discussion in subsequent sections. At the inflow to the DNS $(x / L=-0.60)$, a small but adverse pressure gradient is present. As the flow approaches the bump, the APG strengthens but remains mild (discussed in $\S 3.2$ ), until $x / L=-0.29$ where it switches to a strong FPG accelerating the flow over the upstream side of the bump (discussed in $\S 3.3$ ). At the bump 
(a)

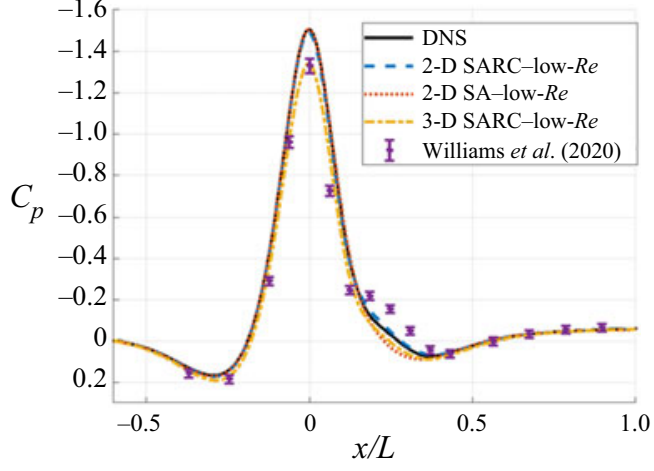

(b)

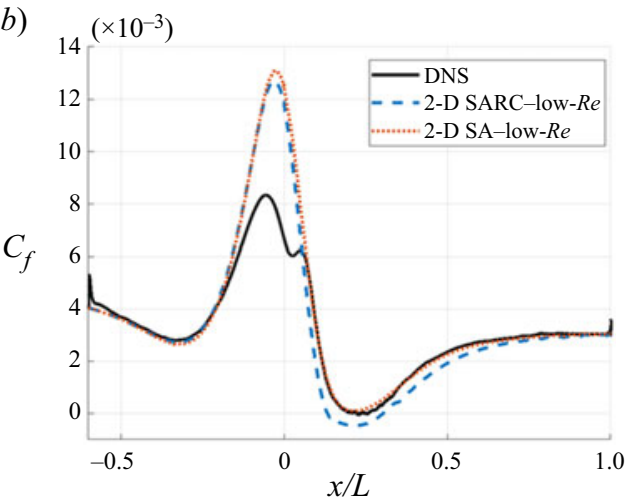

Figure 2. Pressure coefficient $(a)$ and skin friction coefficient $(b)$ on the surface of the 2-D Gaussian bump computed with DNS and 2-D RANS. Pressure data extracted at the centreline from the experiments of Williams et al. (2020) at $R e_{L}=1.37 \times 10^{6}$ and from RANS simulation of the same 3-D geometry at $R e_{L}=1.0 \times 10^{6}$ are also shown for context.

peak, a rapid change from strong FPG to strong APG occurs, with the latter persisting until approximately $x / L=0.40$ (early part discussed in $\S 3.4$ ). Finally, a mild FPG helps the boundary layer recover (not discussed in this paper).

Significant differences are observed among four simulations for the skin friction coefficient predictions in figure 2 (experimental data not available for $C_{f}$ ). Fair agreement is only obtained in the initial mild APG and soon after the start of the strong FPG the curves deviate with RANS largely overpredicting $C_{f}$ over the bump. The DNS solution exhibits a much smaller peak and a local minimum-maximum immediately downstream of the switch from FPG to APG. This feature of the wall shear stress is also present in the DNS of Uzun \& Malik (2020) and is similar to the ones documented for other bump flows with strong FPG (Narasimha \& Sreenivasan 1979; Warnack \& Fernholz 1998; Matai \& Durbin 2019). Of interest is also the streamwise position of the skin friction maximum, which in both cases is located upstream of the bump peak, but occurs farther upstream in the DNS. The size of the separation region is overestimated by RANS as DNS predicts only incipient separation with the mean $C_{f}$ approaching zero $\left(C_{f}<1 \times 10^{-4}\right.$ over $0.19<x / L<0.27$ ) but only becoming negative over a short distance. Instantaneously, however, the flow at the wall does reverse direction and small confined separation bubbles are present.

Contours of the instantaneous vorticity magnitude on the surface of the bump in figure 3 elucidate some of the features of the DNS skin friction coefficient profile. In the APG region upstream of the bump, the footprint of typical near-wall structures (regions of high and low streamwise velocity) is seen as streamwise elongated streaks of high and low vorticity. As the boundary layer progresses through the strong FPG, the acceleration of the flow causes the wall vorticity to rise and the streaks to grow significantly both in width and length. This is a counter-intuitive behaviour and points to the breakdown of the standard relation for the streak spacing $\left(\lambda z \sim 100 v / u_{\tau}\right)$. Obliquely aligned 'puffs' of high turbulent intensity are also visible in this region of the flow, resembling the structures observed in very low-Reynolds-number channel flow by Tsukahara, Seki \& Kawamura (2005). Although the Reynolds number $R e_{\tau}=u_{\tau} \delta / v$ is an order of magnitude larger for the bump, similarities can be drawn between the intermittent state of the turbulence in the channel and the near-wall layer of the strong FPG, as discussed in $\S 3.3$. Towards the end of the FPG, around $x / L=-0.03$, the wall vorticity drops and the streaks become weaker. 


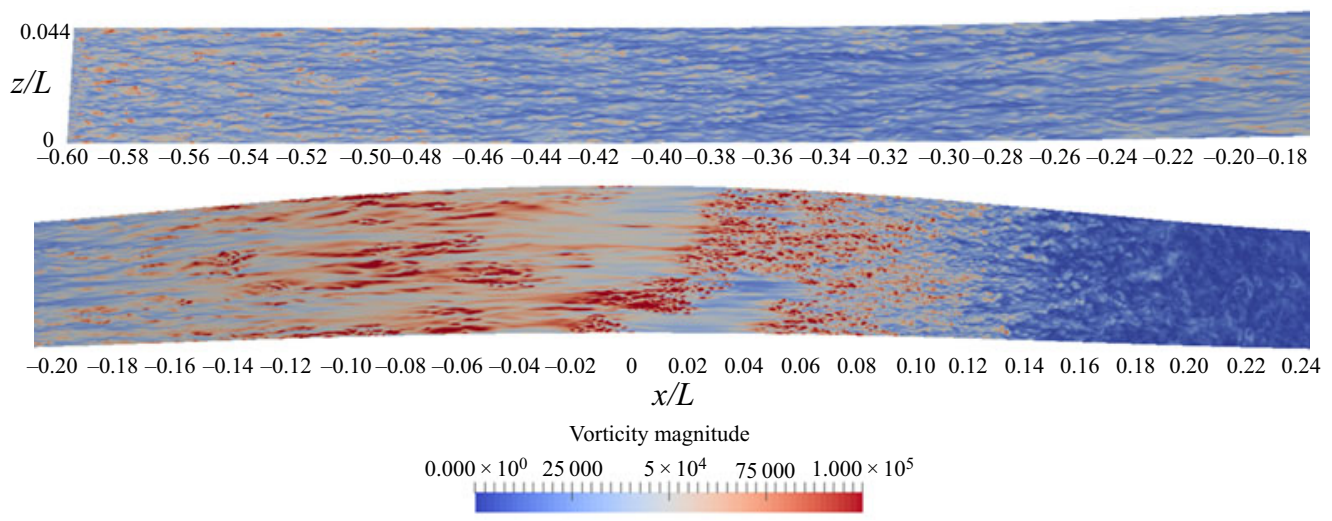

Figure 3. Contours of instantaneous vorticity magnitude on the surface of the Gaussian bump (labelled by normalized streamwise position, wrapped near $x / L=-0.19$ ).

Quiet regions of low vorticity also form. This behaviour explains the steep drop in $C_{f}$ between the maximum value of the curve and the bump peak. Starting slightly upstream of $x / L=0.00$ and continuing into the APG until approximately $x / L=0.05$, spots of large vorticity are found intermittent with the quiet regions, which visually resemble those characteristic of laminar-to-turbulent transition. Note that $x / L=0.05$ is the location of the small local maximum in the skin friction profile. These spots then appear to culminate in a region of intense turbulent activity $(0.06 \leq x / L \leq 0.10)$ with many small-scale and fairly isotropic structures of large vorticity. Continuing farther downstream, the intensity of the small-scale structures decreases as the skin friction also drops. Note that, due to the strong APG which brings the flow to incipient separation, the canonical streaks do not reappear on the downstream side of the bump.

Due to the strong pressure gradients and geometry of the bump, the free stream (irrotational) flow is distorted and highly non-uniform $\left(\partial \bar{u}_{s} / \partial n \neq 0\right)$. As a result, the classical definitions of the boundary layer thickness $\delta 995$ and integral quantities such as the displacement and momentum thicknesses, $\delta^{*}$ and $\theta$, respectively, are no longer applicable. To resolve this issue, the definitions based on the generalized velocity (or 'pseudo-velocity'), $\tilde{U}$, of Spalart \& Watmuff (1993) were used instead. These are repeated in (3.2)-(3.4) from Coleman et al. (2018) for convenience, where $\bar{\omega}_{z}$ is the mean spanwise vorticity - note that the bump-aligned curvilinear coordinate system $(s, n, z)$ is used for the definitions instead of the free stream aligned Cartesian system $(x, y, z)-$

$$
\begin{gathered}
\tilde{U}(s, n) \equiv-\int_{0}^{n} \bar{\omega}_{z}\left(s, n^{\prime}\right) \mathrm{d} n^{\prime} \\
\tilde{\delta}^{*}(s) \equiv \frac{-1}{\tilde{U}_{e}(s)} \int_{0}^{\infty} n \bar{\omega}_{z}(s, n) \mathrm{d} n \\
\tilde{\theta}(s) \equiv \frac{-2}{\left(\tilde{U}_{e}(s)\right)^{2}} \int_{0}^{\infty} n \tilde{U}(s, n) \bar{\omega}_{z}(s, n) \mathrm{d} n-\tilde{\delta}^{*}(s) .
\end{gathered}
$$

The edge velocity in the context of $\tilde{U}$ is defined as

$$
\tilde{U}_{e}(s) \equiv \tilde{U}(s, n \rightarrow \infty),
$$

from which the $99.5 \%$ boundary layer thickness, $\tilde{\delta}_{995}$, is computed as the height above the wall where $\tilde{U}=0.995 \tilde{U}_{e}$. This approach has been used successfully in other studies 


\section{R. Balin and K.E. Jansen}

(a)

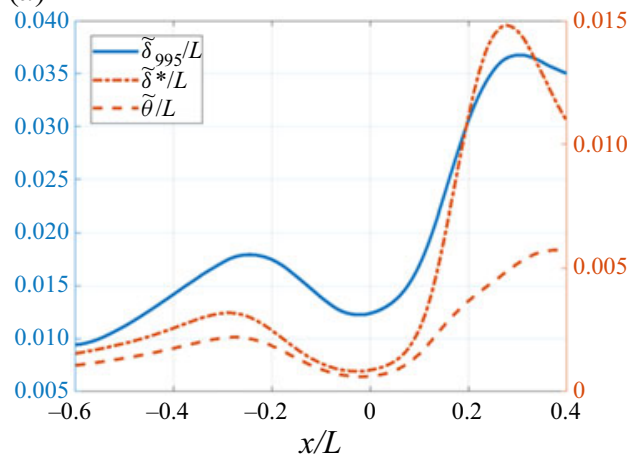

(b)

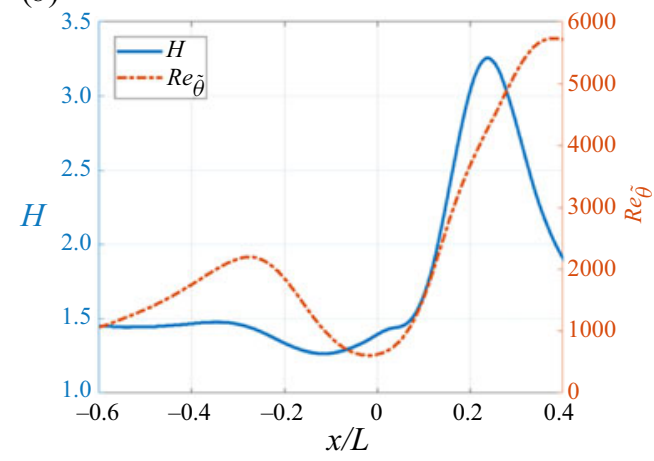

Figure 4. Variation of different boundary layer thickness measures $(a)$ and shape factor $H$ and momentum thickness Reynolds number $\operatorname{Re}_{\tilde{\theta}}(b)$ over the bump.

of pressure gradient flows (Coleman et al. 2018; Uzun \& Malik 2020). For this flow, integration to $n=1.6 \tilde{\delta}_{995}$ resulted in converged profiles for $\tilde{\delta}^{*}, \tilde{\theta}, \tilde{U}_{e}$ and $\tilde{\delta}_{995}$.

Figure 4 shows the variation of the boundary layer integral quantities defined above over the bump surface. In panel $(a)$, the three measures of the boundary layer thickness are presented. The $99.5 \%$ boundary layer thickness generally follows the trends set by the pressure gradients, with a slight delay in its response. In the initial mild APG, $\tilde{\delta}_{995}$ grows until a local maximum at $x / L=-0.25$, which is downstream of the start of the FPG at $x / L=-0.29$. It then shrinks as it progresses through the rest of the FPG reaching a local minimum just upstream of the bump peak. The strong APG on the downstream side of the bump causes $\tilde{\delta}_{995}$ to grow rapidly once again. The displacement thickness follows similar trends, however, it responds more quickly to changes in pressure gradient, as seen by the presence of a local maximum at $x / L=-0.29$ where the pressure gradient changes sign. The third measure of thickness, $\tilde{\theta}$, also behaves similarly to the other two. In this case, the first local maximum is located slightly downstream of the start of the FPG at $x / L=-0.27$, suggesting a slower response to the pressure gradient relative to $\tilde{\delta}^{*}$, but faster than $\tilde{\delta}_{995}$.

Figure $4(b)$ shows the profiles of the shape factor $H=\tilde{\delta}^{*} / \tilde{\theta}$ and $\operatorname{Re}_{\tilde{\theta}}=U_{\infty} \tilde{\theta} / \nu$ over the bump. Soon after the inflow, $H$ settles at a value of 1.44 , which is typical for turbulent boundary layers under weak APG. The initial APG causes the shape factor to rise slightly and form a local maximum at $x / L=-0.35$, which is well upstream of the change in sign of the pressure gradient. Farther downstream, $H$ drops during the initial part of the strong FPG, resulting in a local minimum at $x / L=-0.11$, which is also well upstream of the end of the acceleration. During the remainder of the FPG, $H$ grows gradually and forms a plateau just downstream of the bump peak coinciding with the oscillation in $C_{f}$ observed in figure 2.

In addition to strong pressure gradients, streamline curvature effects are present in this flow. Figure 5 shows the variation of the non-dimensional surface curvature parameters $\hat{\kappa}=\kappa \tilde{\delta}_{995}$ and $\kappa^{+}=\kappa \nu / u_{\tau}$. Additionally, the curvature of a mean flow streamline $\hat{\kappa}_{s l}=$ $\kappa_{S l} \tilde{\delta}_{995}$ located in the outer region of the boundary layer $\left(0.3 \leq n / \tilde{\delta}_{995} \leq 0.8\right)$ is included as a measure of these effects farther from the wall. The similarities between $\hat{\kappa}$ and $\hat{\kappa}_{s l}$ indicate that, in the region of focus of this paper, the outer layer flow is experiencing very similar curvature as the bump surface. As discussed in Baskaran et al. (1991), Narasimha \& Sreenivasan (1979), Schwarz \& Plesniak (1996), So \& Mellor (1973, 1975), due to the significant values of $\hat{\kappa}$ but negligible ones of $\kappa^{+}$, streamline curvature effects are mainly 


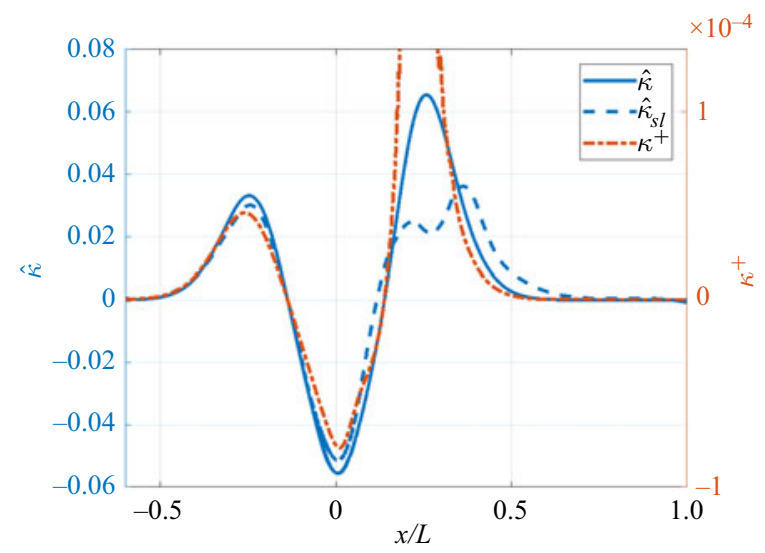

Figure 5. Variation of the non-dimensional surface and streamline curvature of the Gaussian bump. Negative values of $\hat{\kappa}$ and $\kappa^{+}$indicate convex curvature and vice versa.

observed in the outer region of the boundary layer. By contrast, near the wall, direct effects from this disturbance can be considered small, leaving pressure gradient forces to dominate. The interplay between curvature and pressure gradient effects is discussed in the following sections for each portion of the bump flow. Finally, the impact of the Spalart-Shur curvature correction (Spalart \& Shur 1997; Shur et al. 2000) can be seen in figure 2 by comparing the two sets of RANS results. Only a relatively small improvement in $C_{f}$ is observed with the correction in the region of the flow under strong FPG and convex curvature, suggesting that this term is consistent with our assertion that the pressure gradient forces dominate over the curvature near the wall.

The final wall and integral quantities extracted from the DNS are various non-dimensional pressure gradient parameters as shown in figure 6 . These quantities will be discussed more thoroughly in the subsections that follow, however, since they help to characterize the pressure gradients experienced by the flow, they are defined and plotted in this subsection. The acceleration parameter $K$ defined in (1.2) is shown in figure $6(a)$. After a mild APG $\left(K>-1 \times 10^{-6}\right)$ extending from the inflow until $x / L=-0.29$, the strong FPG is visible over the upstream side of the bump. The non-dimensional pressure gradient

$$
\Lambda=-\frac{\partial p}{\partial s} \frac{\tilde{\delta}_{995}}{\tau_{w}},
$$

introduced by Narasimha \& Sreenivasan (1973) and the Clauser pressure gradient parameter

$$
\beta=\frac{\partial p}{\partial s} \frac{\tilde{\delta}^{*}}{\tau_{w}},
$$

are also shown in figure 6(a). Although previous literature has not identified $\beta$ as being particularly illuminating in the context of relaminarization (Narasimha \& Sreenivasan 1979), it is of broad interest in characterizing the strength of the pressure gradients over the bump and is therefore included in the results. Other measures of the pressure gradient and their effects on the near-wall flow are shown in figure $6(b)$. These are $\Delta_{p}$ and $\Delta_{\tau}$ defined in (1.1) and (1.3), respectively. Note that due to the non-dimensionalization by the friction velocity $u_{\tau}$, these two quantities become ill-defined in the vicinity of incipient separation at $x / L=0.19$. 


\section{R. Balin and K.E. Jansen}
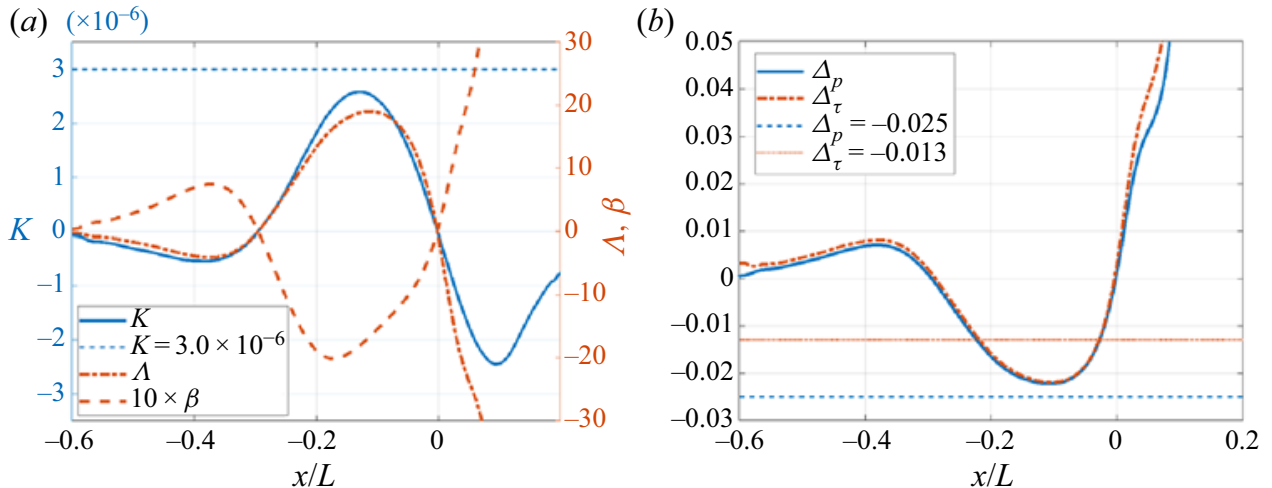

Figure 6. Pressure gradient parameters $K, \Lambda$ and $\beta(a)$ and pressure gradient $\Delta_{p}$ and shear stress gradient $\Delta_{\tau}$ non-dimensionalized by inner units $(b)$ over the bump.

(a)

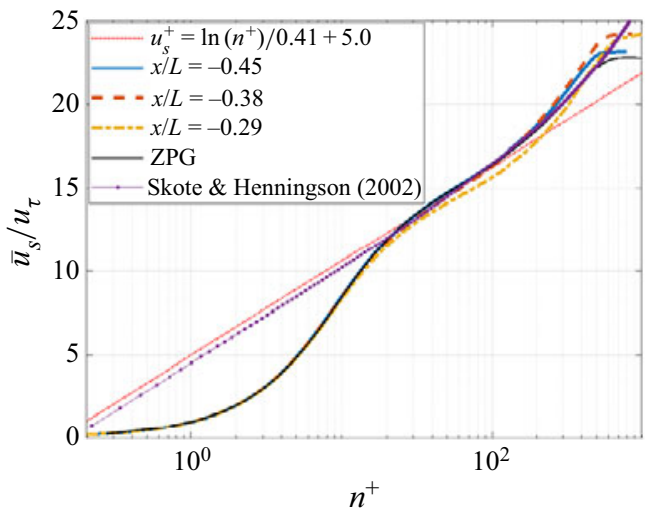

(b)

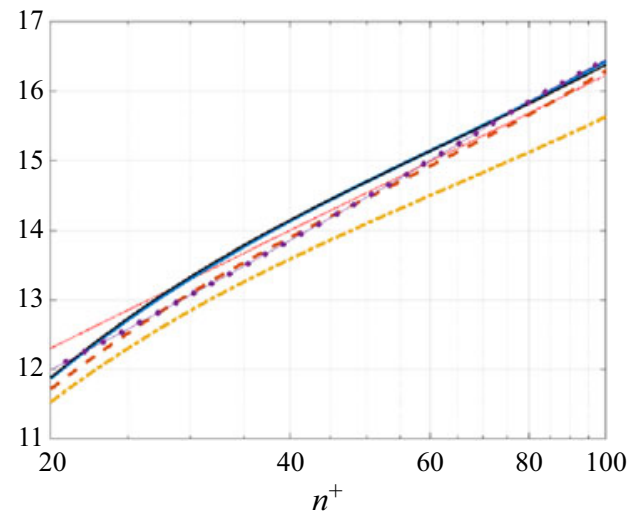

Figure 7. Profiles of the streamwise velocity in the initial mild APG region. Panel $(b)$ zooms into the logarithmic region of panel $(a)$. The ZPG profiles are taken from the DNS of Jimenez et al. (2010) at $\operatorname{Re}_{\theta}=1551$.

With the overall flow field described in terms of the above wall and integral quantities, the following subsections combine this information with profiles of additional mean flow and turbulence quantities, as well as instantaneous contour plots, to analyse and discuss the three pressure gradient regions that are the focus of this paper.

\subsection{Mild APG}

The initial disturbance experienced by the boundary layer is due to a mild APG (a maximum value of $K=-0.5 \times 10^{-6}$ as shown in figure 6) extending from the inflow to the start of the FPG at $x / L=-0.29$. Concave streamline curvature is also present, starting from a negligible amount and gradually increasing throughout this region (see figure 5). Figures 7 and 8 show the development of the streamwise velocity, turbulent kinetic energy (TKE) and Reynolds shear stress in this first section of the bump flow. Note that the TKE is defined as $k=\frac{1}{2}\left(\overline{u_{s}^{\prime 2}}+\overline{u_{n}^{\prime 2}}+\overline{u_{z}^{\prime 2}}\right)$. Additionally, the streamwise location of peak strength of the mild APG is at $x / L=-0.38$ where curvature effects are still growing. 
(a)

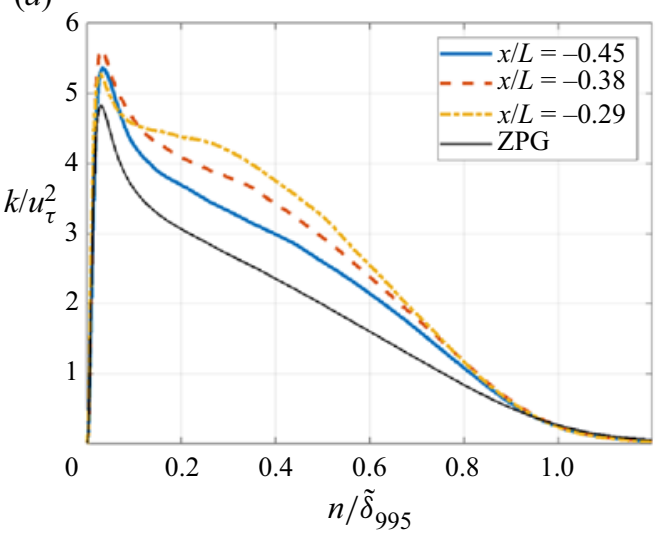

(b)

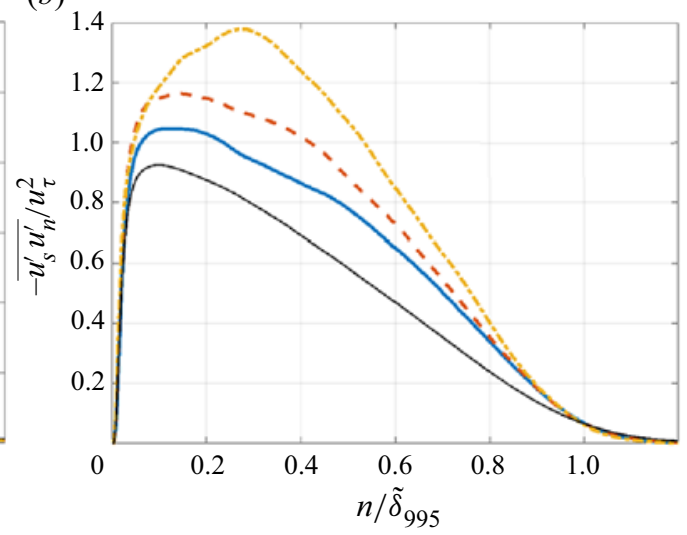

Figure 8. Profiles of the TKE (a) and Reynolds shear stress $(b)$ in the initial mild APG region. The ZPG profiles are taken from the DNS of Jimenez et al. (2010) at $R e_{\theta}=1551$.

In the initial part of this region $(x / L \leq-0.38)$, the APG effects dominate on the velocity, as seen by an increase in the wake region and a slight deviation from the standard logarithmic law. In the remaining portion, however, concave curvature effects dominate in the outer layer by reducing the wake (So \& Mellor 1975), while the logarithmic region is shifted farther below the standard law. It is interesting to point out from the zoomed-in view in figure $7(b)$ that at $x / L=-0.45$ the logarithmic region is in great agreement with the ZPG profile of Jimenez et al. (2010), whereas the slight shift at $x / L=-0.38$ is accurately predicted by the scaling law of Skote \& Henningson (2002)

$$
u_{s}^{+}=\frac{1}{\kappa}\left(\ln \left(n^{+}\right)-2 \ln \left(\frac{\sqrt{1+\gamma n^{+}}+1}{2}\right)+2\left(\sqrt{1+\gamma n^{+}}-1\right)\right)+B,
$$

where $\kappa=0.41, B=4.5, \gamma=\left(u_{p} / u_{\tau}\right)^{3}$ and $u_{p}=((v / \rho) \partial p / \partial s)^{1 / 3}$. Note that, while the accumulated action of the APG is more visible at $x / L=-0.29$ due to the significant shift below the standard logarithmic law (Skote \& Henningson 2002), the scaling in (3.8) would not suggest any correction in the slope at this location due to the pressure gradient being zero. Both the non-dimensional TKE and Reynolds shear stress increase throughout this region of the flow, particularly in the outer layer at $n / \tilde{\delta}_{995} \approx 0.3$, which is consistent with APG and concave curvature effects (So \& Mellor 1975; Kitsios et al. 2016).

Figures 7 and 8 show that while the initial pressure gradient is only mild, its effects combined with the concave curvature cause significant changes to the boundary layer structure. Consequently, the boundary layer entering the strong FPG farther downstream is not the canonical ZPG flat plate boundary layer. This feature distinguishes this bump flow from many of the experimental studies performed on strong FPG effects and relaminarization which employed a monotonic acceleration from a ZPG state.

\subsection{Strong FPG}

This section focuses on the FPG region of the Gaussian bump $(-0.29 \leq x / L \leq 0.00)$ and describes the effects of this force on the mean flow and turbulence. The interplay between the FPG and streamline curvature is also discussed, which, as shown in figure 5, is both concave and convex over this section of the flow. However, before presenting the results 


\section{R. Balin and K.E. Jansen}

of this study and to ground the observations that follow in the available body of literature, it is helpful to review the effects of strong FPG on turbulent boundary layers more deeply than the background provided in the introduction.

When a strong FPG is applied and removed, a turbulent boundary layer undergoes three successive stages that significantly change its fundamental characteristics (Sreenivasan 1982). Soon after the start of the acceleration, the boundary layer exhibits significant departures from the standard laws while remaining in a fully turbulent state. Following the terminology of Sreenivasan (1982), a boundary layer in this first stage is said to be laminarescent. It can be considered a precursor to relaminarization, however, it does not guarantee the onset of the process. Some of the key signs of laminarescence are a deviation of the streamwise velocity from the standard logarithmic law (first below and then above Patel \& Head (1968)), significant growth of the viscous sublayer in local wall units, a reduction in the $99.5 \%$ boundary layer thickness, and a reduction in the rate of wall-layer bursting.

Experiencing further sustained acceleration, the boundary layer enters a relaminarization process. This is the second stage, and lasts until the process is complete resulting in a quasi-laminar boundary layer. It is important to highlight that this is a gradual process, thus the onset does not imply that a quasi-laminar state is achieved. During relaminarization, the previously fully turbulent boundary layer develops a viscous dominated inner layer stabilized by the acceleration, while the turbulence in the outer layer is distorted (Sreenivasan 1982). Moreover, the skin friction is observed to drop while the flow still accelerates, the shape factor increases, and the relative (non-dimensionalized by local $u_{\tau}$ ) Reynolds stresses and TKE production drop significantly.

The final stage occurs when the FPG is relaxed. The acceleration can no longer stabilize the near-wall flow, and soon after the onset of instability a retransition process originating near the wall returns the boundary layer towards a fully turbulent state. This process is rapid and shares some similarities to laminar-to-turbulent transition, such as the formation and growth of turbulent spots (Blackwelder \& Kovasznay 1972). Other notable features of this process are a sudden rise in skin friction, a significant enhancement of the turbulent intensities, and a local maximum of the shape factor.

Since relaminarization is a process that brings about significant changes to the boundary layer, it is of great interest to identify the onset and completion points. The latter can be easily defined as the location where the effects of the Reynolds stresses on the mean flow dynamics are negligible. Fluctuations are still present as a remnant from the upstream flow, but do not contribute to the development of the mean velocity. The near-wall bursting process has also ceased, therefore eliminating production of TKE (Narasimha \& Sreenivasan 1979; Sreenivasan 1982). The onset point, however, is more complicated to define and identify, yet it is extremely relevant for turbulence models designed to predict this phenomenon. The remainder of this section discusses the laminarescent and relaminarization stages of the boundary layer over the Gaussian bump and draws comparisons with the non-dimensional parameters and methods proposed in the literature.

Conclusive evidence of the initiation of the relaminarization process taking place in the near-wall region of the boundary layer over the Gaussian bump is provided by the instantaneous contours of vorticity in figure 9. The figure shows instantaneous vortical structures at a few locations of constant $n / \tilde{\delta}_{995}$. Note that because $\tilde{\delta}_{995}$ varies with downstream position, these surfaces track the local boundary layer height, not distance to wall. The vorticity magnitude is normalized by the local (same streamwise location) timeand spanwise-averaged wall vorticity magnitude $\bar{\omega}_{w}$ in order to remove the increase of vorticity due to flow acceleration and highlight the fluctuations relative to their local mean wall value. Moreover, solid black lines across the width are placed to define important 


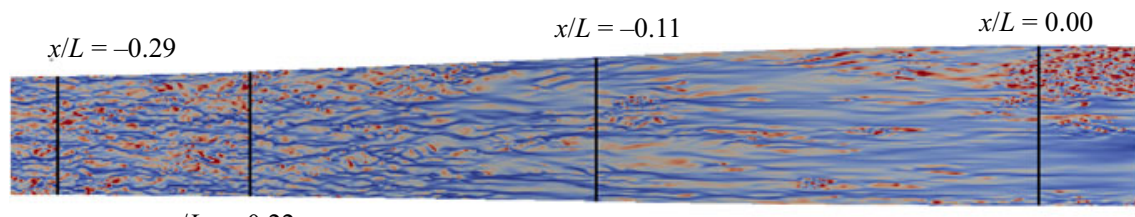

$x / L=-0.22$

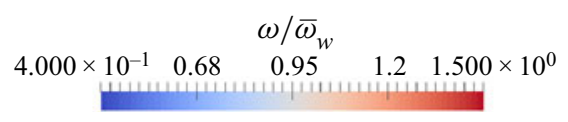
$x / L=-0.29$
$x / L=-0.11$
$x / L=0.00$

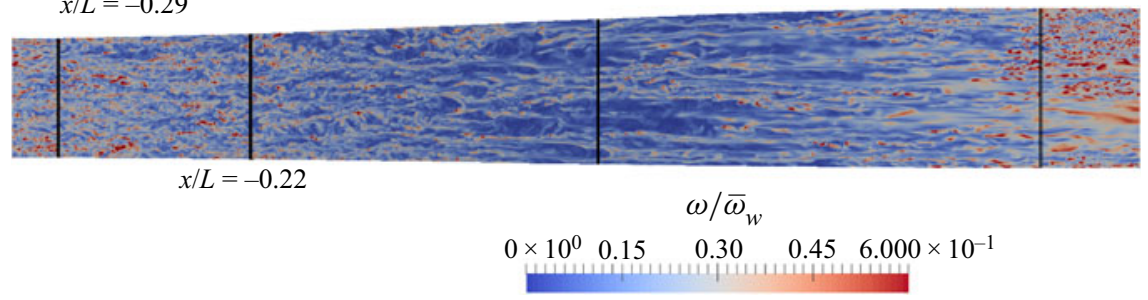

$x / L=-0.29$

$x / L=-0.11$

$x / L=0.00$

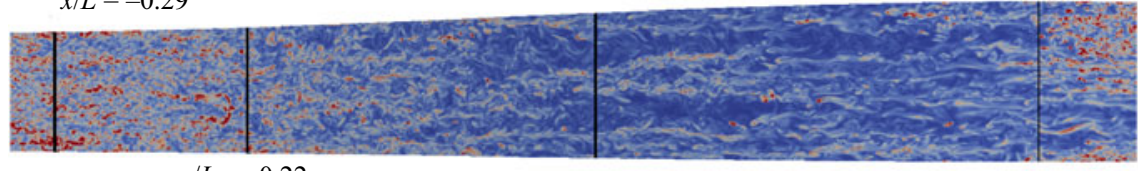

$x / L=-0.22$

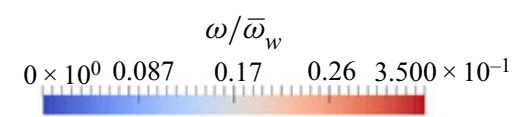

$x / L=-0.11 \quad x / L=0.00$

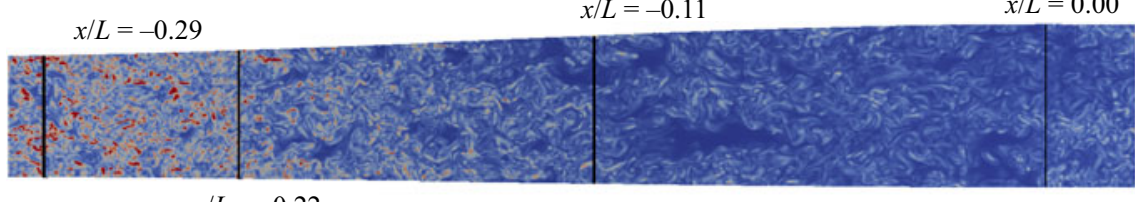

$x / L=-0.22$

$$
\begin{aligned}
& \omega / \bar{\omega}_{w} \\
& \begin{array}{lllll}
0 \times 10^{0} & 0.062 & 0.12 & 0.19 & 2.500 \times 10^{-1}
\end{array}
\end{aligned}
$$

Figure 9. Instantaneous vorticity magnitude normalized by the local time- and spanwise-averaged wall vorticity at different locations within the boundary layer in the FPG region of the bump. From top to bottom, the heights above the wall of the slices are $n / \tilde{\delta}_{995}=0.01,0.05,0.1,0.4$. The black vertical lines mark the streamwise location of key events of the flow.

landmarks within the FPG region. These are: the start of the FPG at $x / L=-0.29$; the location where the streamwise velocity departs above the logarithmic law at $x / L=-0.22$ (discussed in more detail later); the peak strength of the FPG at $x / L=-0.11$; and the bump peak and end of the FPG at $x / L=0.00$. Note that the same figures were also analysed at different time steps in order to confirm that the following features are always present and not just at the time instant shown here.

Very close to the wall, the typical streamwise streaks of high and low vorticity are visible at the beginning of the FPG region. These remain fairly unchanged until around $x / L=$ -0.22 , downstream of which the streaks appear to stretch in the streamwise direction and reduce in relative intensity. As the location of peak FPG strength is approached, the character of the fluctuations is significantly altered. The streaks become very elongated in 


\section{R. Balin and K.E. Jansen}

the streamwise direction while also thickening in the spanwise direction, and their relative intensity is significantly reduced. Similar observations were reported in the experiments of Volino (2020). These changes continue past the peak FPG until the peak of the bump, where quiet flow with weak fluctuations surround a few regions with smaller and more intense scales.

Slightly farther from the wall, at $n / \tilde{\delta}_{995}=0.05$ and 0.1 , similar trends can be observed. Fully turbulent flow is present at the start of the FPG and is maintained until slightly downstream of $x / L=-0.22$, after which the turbulent scales weaken relative to the wall vorticity, stretch significantly in the streamwise direction, and quiet regions of low vorticity appear. At this height above the wall, the streaks of high vorticity fluctuations turn into very thin and long ridges, while the quiet areas form 'valleys' that are much wider but equally as long as the newly formed ridges. These are clear signs that the near-wall region of the boundary layer is no longer fully turbulent, and instead becomes intermittent and approaches a quasi-laminar state. A relaminarization process is therefore taking place in this flow. The contours also show how this truly is a gradual process since no clear demarcation can be identified for the start of intermittency. However, although it cannot be confidently discerned from the contours in figure 9, it will be shown later in this section that the process does not complete and a quasi-laminar state is not achieved by the end of the FPG region, thus making this only a partial relaminarization.

In the outer layer, at $n / \tilde{\delta}_{995}=0.4$, the turbulence is also very much affected by the strong FPG. As the flow is being accelerated, the normalized vorticity fluctuations weaken with the smaller scales decaying entirely while the larger scales remain fairly constant in size and shape. This disappearance of the rich small scales is consistent with the decrease in $\operatorname{Re}_{\tilde{\theta}}$ and the stabilizing effects of the FPG and convex curvature. Additional insight would be gained from the energy spectra of the velocity fluctuations, however, this exercise is left as future work.

As was noted earlier, both concave and convex streamline curvature effects are acting during the FPG, with the change from the former to the latter being located at $x / L=$ -0.14 . The vorticity contours in figure 9 clearly show that changes to both the near-wall and outer layer turbulence structures are taking place where the destabilizing concave curvature is acting, well before the stabilizing convex curvature takes effect. In fact, some of the larger 'valleys' of quiet and low relative vorticity flow appear upstream of the change in sign of the surface curvature at around $x / L=-0.15$. Moreover, intermittency is limited to the near-wall region, where curvature effects measured by $\kappa^{+}$are small (see figure 5). The pressure gradient effects are therefore deemed the dominant force responsible for the onset of relaminarization over the Gaussian bump, rather than the combination of pressure and curvature effects.

Further evidence of a relaminarization process taking place due to the strong FPG is found in the skin friction profile shown in figure 2. First, the maximum $C_{f}$ is located significantly upstream of the bump peak at $x / L=-0.055$ where the flow is still experiencing a fairly strong acceleration $\left(K=1.7 \times 10^{-6}\right.$ from figure 6$)$. Second, the sudden rise in skin friction at the start of the APG forming the local minimum and maximum in quick succession, along with the appearance of high-intensity turbulent spots at the bump peak as seen in figures 3 and 9, indicate the presence of partial retransition. The details of this process are discussed in $\S 3.4$, however, it implies that the upstream boundary layer does not remain fully turbulent and is significantly weakened by the FPG. Both of these behaviours are key characteristics of relaminarizing boundary layers (Narasimha \& Sreenivasan 1973; Sreenivasan 1982; Warnack \& Fernholz 1998) and are in contrast to what is expected of a fully turbulent flow under the same pressure gradient, which resembles more closely the RANS prediction in figure 2 . 


\section{DNS of a boundary layer with strong pressure gradients}

Having established the presence of a partial relaminarization process over the upstream side of the bump, as urged by Sreenivasan (1982), it is important to discuss the various pressure gradient parameters used to determine the onset of relaminarization by previous experiments discussed in the literature. Figure 6 presents a number of these for the region of the flow this paper focuses on. In this region, $K$ (defined in (1.2)) approaches, but does not cross, the limit of $K=3.0 \times 10^{-6}$ used to mark the start of a relaminarization process in previous studies (Narasimha \& Sreenivasan 1979; Sreenivasan 1982; Spalart 1986). The maximum value is in fact $2.58 \times 10^{-6}$ at $x / L=-0.13$, which is still below the range $2.75 \times 10^{-6} \leq K \leq 3.00 \times 10^{-6}$ identified with DNS of a series of sink flows (Spalart 1986). Therefore, the acceleration parameter is not an accurate predictor of the onset of relaminarization for this flow, especially considering that figure 9 shows large intermittent regions appearing in the near-wall region around $x / L=-0.15$ where $K=2.51 \times 10^{-6}$. This result is, however, consistent with criticisms about the unsuitability of $K$ for predicting near-wall physics discussed in previous studies (Patel \& Head 1968; Sreenivasan 1982).

The non-dimensional pressure gradient, $\Lambda$ (defined in (3.6)), introduced by Narasimha $\&$ Sreenivasan (1973) is also shown in figure 6(a). Values above 50 have been proposed as a sign of the completion of the process and the achievement of a quasi-laminar state (Narasimha \& Sreenivasan 1973, 1979; Sreenivasan 1982). Furthermore, based on the data presented in these studies, values of $\Lambda$ between 10 and 25 can be correlated to the departure from fully turbulent flow and thus the onset of relaminarization. The maximum value obtained by the FPG is around 19, indicating that $\Lambda$ can be a useful indicator of the start of the process for this flow as well as supporting the statement that a quasi-laminar state or completion of the relaminarization process is not achieved.

From figure $6(b)$, similar to the profile for the acceleration parameter $K$, the boundary layer over the bump does not reach the generally accepted critical value of $\Delta_{p}=-0.025$ identified to mark the onset of relaminarization (Narasimha \& Sreenivasan 1979; Spalart 1986). The minimum value is $\Delta_{p}=-0.022$ at $x / L=-0.11$, whereas $\Delta_{p}=-0.021$ at $x / L=-0.15$. These lower magnitudes are supported by the work of Narayanan $\&$ Ramjee (1969), who reported -0.020 as the critical number. It is also worth mentioning that the initial study by Patel (1965) even suggested -0.018 , although this was only a tentative value and was later updated to -0.024 (Patel \& Head 1968). With regards to the shear stress gradient parameter $\Delta_{\tau}$ in figure $6(b)$, the critical value of interest is -0.013 , which has been used to mark the start of the deviation above the logarithmic law (Patel \& Head 1968; Bradshaw 1969). This phenomenon is discussed in detail later in this section. The boundary layer over the bump reaches $\Delta_{\tau}=-0.013$ relatively soon after the start of the FPG at $x / L=-0.22$.

Finally, integral quantities, such as the shape factor $H$ and similar ratios (Cal \& Castillo 2008), have also been investigated for strongly accelerated flows in connection to the onset of relaminarization (Blackwelder \& Kovasznay 1972; Narasimha \& Sreenivasan 1973; Warnack \& Fernholz 1998). However, for the boundary layer over the Gaussian bump, these parameters were not insightful in describing the start or even the presence of this process. This result is not surprising given the discussion regarding the uncertainties associated with using $H$ for this purpose in Sreenivasan (1982).

The above discussion highlights that, while contours of instantaneous vorticity clearly show the presence of a partial relaminarization process due to the strong FPG, most of the guidelines offered by previous studies based on various pressure gradient parameters and integral quantities were not reliable indicators of the onset of relaminarization for this bump flow. The disagreement with previous studies is likely caused by the added complexities of this flow. The literature mainly focused on equilibrium sink flows or 

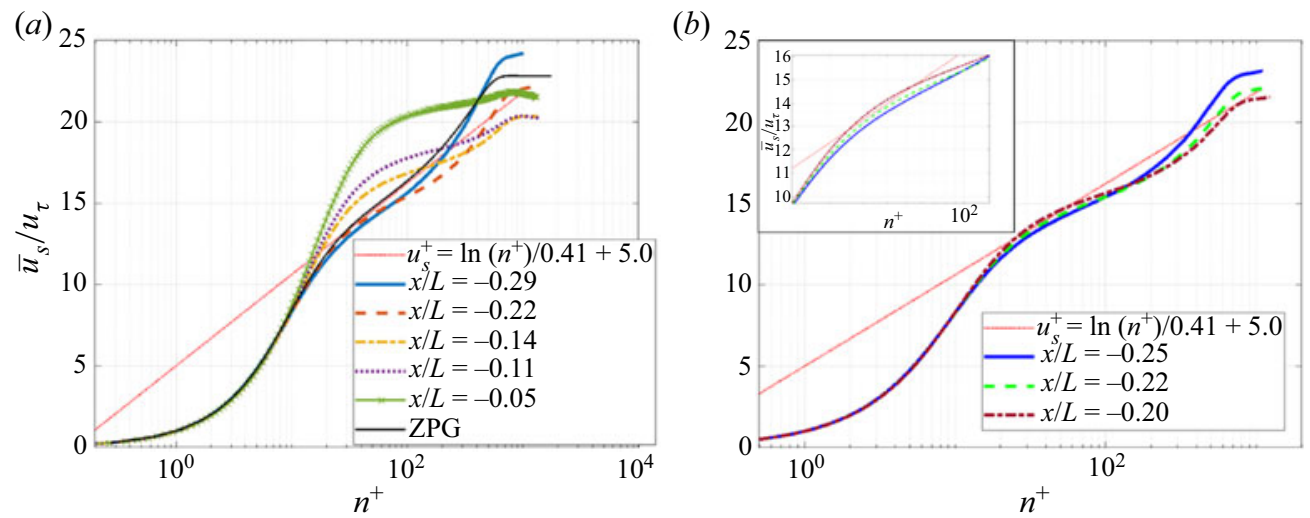

Figure 10. Mean streamwise velocity profiles in the FPG region normalized by wall units. The ZPG profiles are taken from the DNS of Jimenez et al. (2010) at $R e_{\theta}=1551$.

boundary layers over flat plates that were monotonically accelerated from a zero pressure gradient by variation of the top boundary condition. The boundary layer over the Gaussian bump is deeply different; it experiences an APG upstream of the acceleration which, while being mild, affects the velocity and stress profiles in a significant manner, there is a high degree of non-equilibrium in the sense of non-constant Clauser pressure gradient parameter, streamline curvature effects are present throughout the entire flow, and the Reynolds number is lower than what most experimental studies were able to achieve. Furthermore, given the results discussed above, a definite marker or critical value for the onset of relaminarization was not found. Only an approximate location of $x / L=-0.15$ was offered based on the contours in figure 9. This is not a surprising conclusion, and in fact agrees with the interpretation in Sreenivasan (1982) and Warnack \& Fernholz (1998). By extension, this study provides further evidence that critical parameters of any specific quantity should only be used as guidelines to suggest that relaminarization might be occurring, but further analysis of the state of the turbulence is needed for a more definite statement to be made. Moreover, a turbulence model based on the $K$ and $\Delta_{p}$ parameters is likely to not be universal.

Figure 10(a) shows the non-dimensional streamwise velocity profile at a number of locations on the upstream side of the bump. At the start of the FPG $(x / L=-0.29)$, the velocity shows a logarithmic region shifted slightly below the standard values $(\kappa=0.41$ and $B=5.0$ ) consistent with the mild APG effects discussed in the previous section. Soon after that, at $x / L=-0.22$, the wake is significantly reduced and the profile lacks a linear region that would result from a logarithmic relationship. Therefore, there is a breakdown of the logarithmic law with the velocity remaining for the most part below the law. As the flow progresses through the FPG, the effects become increasingly stronger. The velocity continues to rise above the logarithmic law until the peak of the bump, with a thickening of the viscous sublayer and a continuous reduction of the wake in the outer layer. Note that convex curvature effects are also present downstream of $x / L=-0.14$, however, the continued reduction of the wake downstream of this location indicate that the pressure gradient is dominating over curvature on the velocity. This result is consistent with the experimental study of Schwarz \& Plesniak (1996).

Additionally, figure $10(b)$ shows the velocity profiles at three locations in the proximity of $x / L=-0.22$ where the boundary layer achieves the critical parameter $\Delta_{\tau}=-0.013$ (see figure 6). The profiles cross the standard logarithmic law right at the critical value, 


\section{DNS of a boundary layer with strong pressure gradients}

providing striking agreement with Bradshaw (1969) and the notion of Patel \& Head (1968) that the non-dimensional shear stress gradient is a suitable quantity to measure the departure above the logarithmic law. Following the description in Sreenivasan (1982), this behaviour is a clear indicator of the laminarescent boundary layer that precedes relaminarization. Regardless of the nomenclature used, when considered along with the non-dimensional vorticity contours in figure 9, the velocity profiles provide further evidence that: (1) strong FPG cause the breakdown of the standard logarithmic law and a departure above it while the flow is still fully turbulent; (2) the breakdown of these standard laws comes with a change in the fundamental character of the turbulence (significant changes in the vortical structures in figure 9 can be seen to gradually occur across the $x / L=-0.22$ line at all values of $n / \tilde{\delta}_{995}$ ); and (3) these changes to the velocity occur at much smaller values of the non-dimensional pressure gradients relative to the critical ones $\left(K=1.4 \times 10^{-6}\right.$ where $\left.\Delta_{p}=\Delta_{\tau}=-0.013\right)$, and farther upstream than evidence of intermittency in the near-wall turbulence, and thus these alone should not be considered a sign of the onset of relaminarization.

Reynolds stress and TKE profiles in the FPG region are shown in figure 11 normalized by the free stream velocity $U_{\infty}$. The streamwise fluctuations develop a large inner peak and an outer knee point, which progressively increases and decreases, respectively, as the turbulence advects through the pressure gradient. Not surprisingly, the TKE follows very similar trends. Note that the increase of the streamwise Reynolds stress near the wall is consistent with the instantaneous contours of vorticity in figure 9 and the acceleration of the flow. The wall-normal fluctuations $\overline{u_{n}^{\prime 2}}$ show an increase in intensity in the initial part of the FPG which is followed by a significant reduction. The spanwise Reynolds stress behaves similarly, with only a slight change at $x / L=-0.22$, followed by a decrease farther downstream. The shear stress $-\overline{u_{s}^{\prime} u_{n}^{\prime}}$ exhibits interesting features. At $x / L=-0.22$, the outer layer shows an increase in the stress, however, near the wall, the profile shows a sudden 'break' (more visible in figure $12 b$ as a sudden change in the slope at $n / \tilde{\delta}_{995}=0.03$ and $\left.-\overline{u_{s}^{\prime} u_{n}^{\prime}} / u_{\tau}=0.7\right)$, indicating a change in the near-wall turbulence. Downstream of this location, the profiles develop a bimodal shape, with an inner peak which appears to be relatively fixed in magnitude and distance from the wall $\left(n / \tilde{\delta}_{995}=0.04\right)$, and an outer peak which continuously decreases and moves farther from the wall.

The appearance of knee points and multiple peaks in the Reynolds stress profiles is indicative of an internal layer being present in the flow. This is a common feature of boundary layers over bumps and hills. Previous studies identified the cause of these internal layers to be due to a sudden change in the wall boundary conditions and thus shear stress, including changes in pressure gradients (Tsuji \& Morikawa 1976) and surface curvature discontinuities (Baskaran et al. 1987; Webster et al. 1996; Wu \& Squires 1998). Since the Gaussian shape of the bump ensures continuity of the curvature, the internal layer observed here is caused by the change in pressure gradient from adverse to favourable at $x / L=-0.29$. Moreover, in the presence of internal layers, the inner and outer layers become almost independent of each other, with the latter behaving similarly to a free shear layer. Similar behaviour in the presence of internal layers was also reported by Baskaran et al. (1987); by Antonia \& Luxton $(1971,1972)$ due to sudden changes in surface roughness; and by Abe (2020) due to sudden imposition of a surface spanwise velocity. The internal layer has a fairly constant Reynolds shear stress throughout the FPG as suggested by the small variation in the magnitude of the inner peak in figure $11(d)$. This feature was noted for other strongly accelerated flows (Narasimha \& Sreenivasan 1979). Additionally, the internal layer shows a rapidly evolving anisotropy of the normal stresses in favour of 

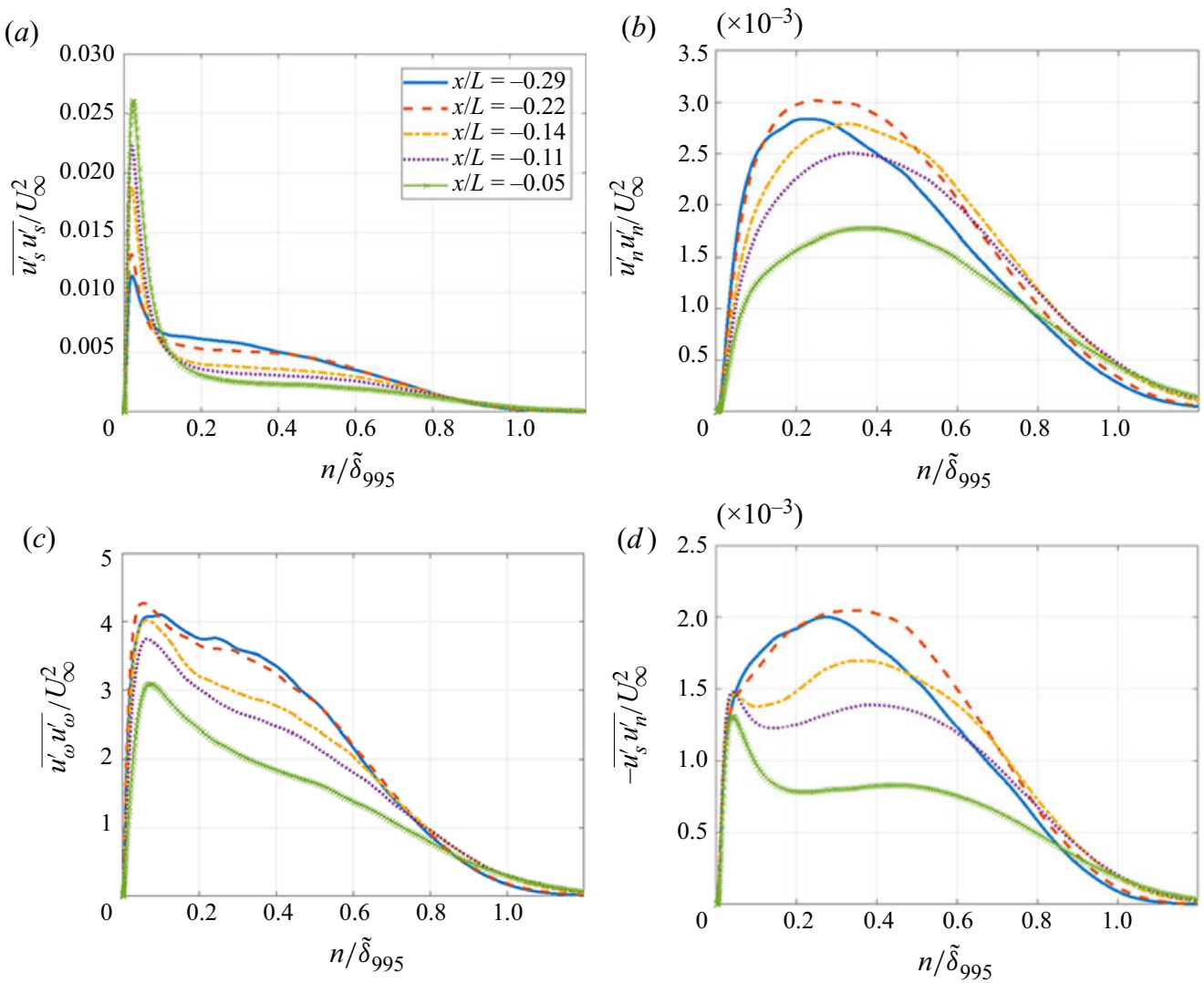

(e)

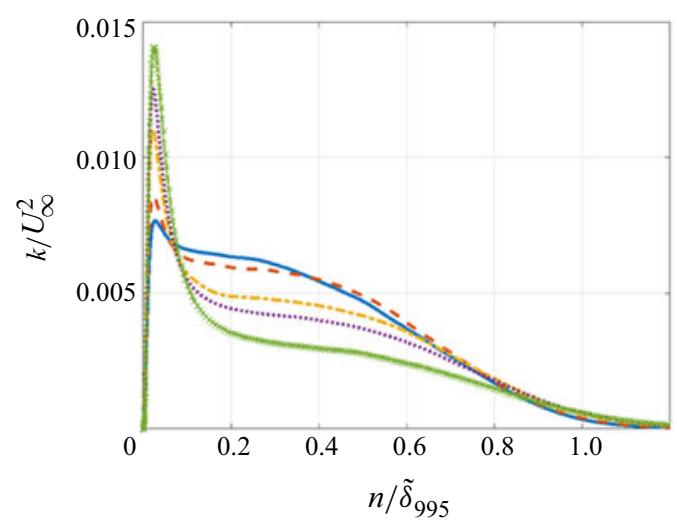

Figure 11. Mean Reynolds stresses and TKE in the FPG region of the Gaussian bump normalized by $U_{\infty}$.

the streamwise direction and at the expense of the wall-normal direction predominantly. This behaviour is consistent with the instantaneous contours of vorticity in figure 9 .

Moreover, a closer look along the initial part of the FPG $(-0.29 \leq x / L \leq-0.22)$ reveals that the 'break' in the shear stress profile near the wall appears as soon as the pressure gradient changes sign. Similarly, the near-wall peak of the TKE starts to grow immediately downstream of $x / L=-0.29$. Since the curvature changes direction approximately halfway along the FPG, these two inner layer effects appear to be strongly 
(a)

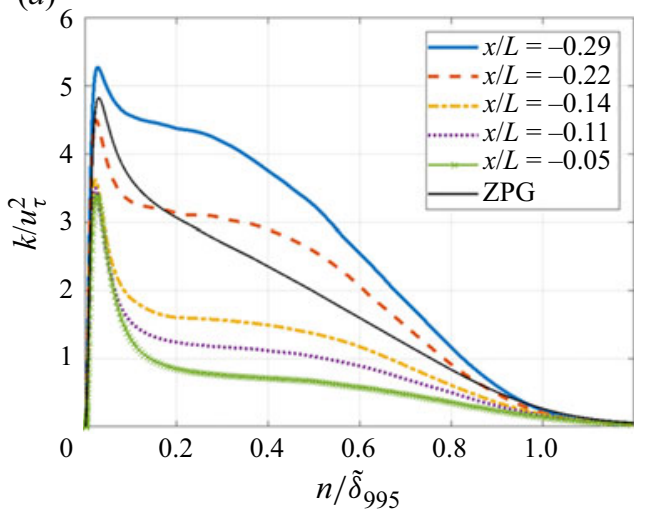

(b)

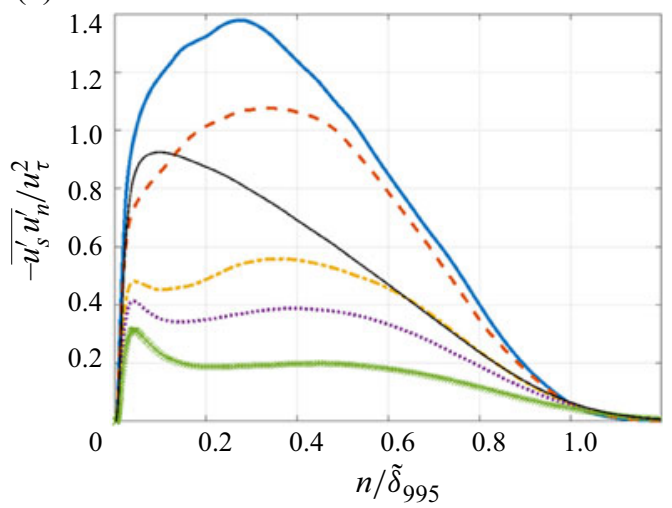

(c)

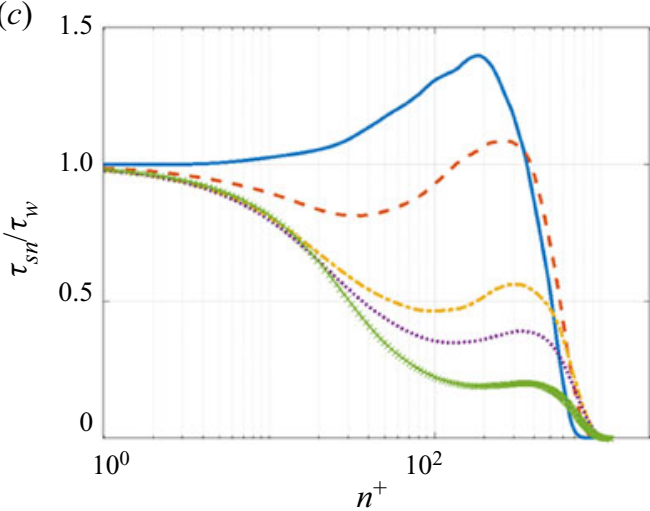

Figure 12. Mean TKE (a), Reynolds shear stress $(b)$ and total shear stress $(c)$ in the FPG region of the Gaussian bump normalized by wall units. The ZPG profiles are taken from the DNS of Jimenez et al. (2010) at $R_{\theta}=$ 1551 .

related to the pressure gradient rather than the curvature. In the outer layer, the behaviour of the Reynolds stresses is slightly more complex during this initial phase, with the inactive components (streamwise and spanwise) behaving differently than the active (shear and wall-normal) ones. The former remain fairly unchanged, while the latter follow the trends observed in the streamwise velocity in figure 10 . The effects of the significant concave curvature dominate over the FPG at $x / L=-0.22$, increasing these two components of the stress. Farther downstream, as the concavity is reduced and the strength of the FPG increases, pressure gradient effects dominate and all components of the stress tensor decrease significantly. This response to the FPG in the outer region of the boundary layer is in great agreement with the recent experiments of Volino (2020).

This analysis, particularly of the shear stress, strongly suggests the independence of the inner and outer layers. The former is dominated by FPG effects, while the latter responds to a combination of streamline curvature and pressure gradient. It is the inner layer physics, however, that are mostly responsible for the skin friction coefficient over the bump. Consequently, a turbulence model that hopes to be predictive of a flow of this kind must be able to capture these near-wall effects. Note that the Gaussian bump flow considered here is not an isolated test case of these effects, and similar examples exist in the literature (Wu \& Squires 1998; Uzun \& Malik 2018; Matai \& Durbin 2019). 


\section{R. Balin and K.E. Jansen}

Further interesting observations arise from the TKE and Reynolds shear stress profiles normalized by wall units in figure 12. This non-dimensionalization is useful to show changes in these quantities relative to the local wall shear rather than a comparison of their absolute magnitudes. Additionally, note that $\tau_{s n}$ represents the total shear stress, which is the sum of the Reynolds and viscous stresses. As the turbulence advects through the FPG, the wall-shear normalized TKE is significantly reduced everywhere across the boundary layer, including at the peak. This trend of the near-wall TKE is in contrast to the one shown in figure 11, and indicates that while the streamwise fluctuations are strengthening near the wall as the flow is being accelerated, they are not increasing fast enough relative to the increase in wall shear stress. Moreover, the drop in TKE in the outer layer is magnified with this non-dimensionalization. These trends are in full agreement with the vorticity contours in figure 9 given the similar non-dimensionalization used. The Reynolds shear stress exhibits similar trends to the TKE showing significant reduction due to the FPG. In this non-dimensional form, the inner peak is not constant and instead continues to diminish reaching values as low as $35 \%$ of the wall shear stress (a density of one was used in the simulation resulting in $u_{\tau}^{2}=\tau_{w}$ ).

The non-dimensional total shear stress in figure 12 clearly shows the significant drop in turbulent stress relative to the viscous stress both in the inner and outer layers. Considering both the Reynolds and total shear stress profiles in the figure, at $x / L=-0.05$ the viscous stress dominates over the turbulent one until approximately $n^{+}=50$, clearly pointing to a thickening of the viscous sublayer measured in wall units. This in turn leads to a very steep wall-normal gradient at a distance between $10 \leq n^{+} \leq 60$, which directly impacts the streamwise momentum equation and thus the streamwise velocity as seen in figure 10 . In other words, it is the steep gradient in the total shear stress that is responsible for the deviation above the logarithmic law. Once this gradient reaches a certain magnitude, measured in part by $\Delta_{\tau}$, the breakdown of the logarithmic law is to be expected. Note that while the relative size of the Reynolds shear stress is significantly reduced by the FPG, it is not negligible and thus the relaminarization process does not reach completion and the flow is still turbulent, albeit only intermittently in the near-wall region. The outer layer, by contrast, remains fully turbulent with the fluctuations decaying in intensity.

It is also of interest to discuss changes to the correlation coefficient, which is defined as

$$
C_{\tau}=\frac{-\overline{u_{s}^{\prime} u_{n}^{\prime}}}{\sqrt{\overline{u_{s}^{\prime 2}}} \sqrt{\overline{u_{n}^{\prime 2}}}},
$$

and is plotted in figure 13 for the same streamwise locations along the FPG. At $x / L=$ -0.29 , the coefficient has the canonical value of 0.5 in the outer layer, and slightly lower near the wall. Farther downstream, in the region $-0.29<x / L \leq-0.14, C_{\tau}$ increases everywhere within the boundary layer. Given the significant strength of the FPG in this region, this change is attributed to the concave curvature of the mean streamlines $(\hat{\kappa}$ in figure 5 is a maximum at $x / L=-0.24$ ). Only downstream of $x / L=-0.14$, where the curvature changes to convex, $C_{\tau}$ decreases rapidly as the boundary layer approaches the bump peak. These results are in agreement with Narasimha \& Sreenivasan (1979), Spalart (1986) and So \& Mellor (1973), and support previous observations that the strong FPG does not change the correlation between these two turbulent fluctuations, even though the Reynolds stresses are significantly reduced and the turbulence character is significantly altered during relaminarization.

Finally, useful quantities for the analysis of the boundary layer and for evaluating and improving turbulence models are the production rates for the TKE and the Reynolds shear stress. These quantities are shown in figure 14 for a number of stations along the FPG and 


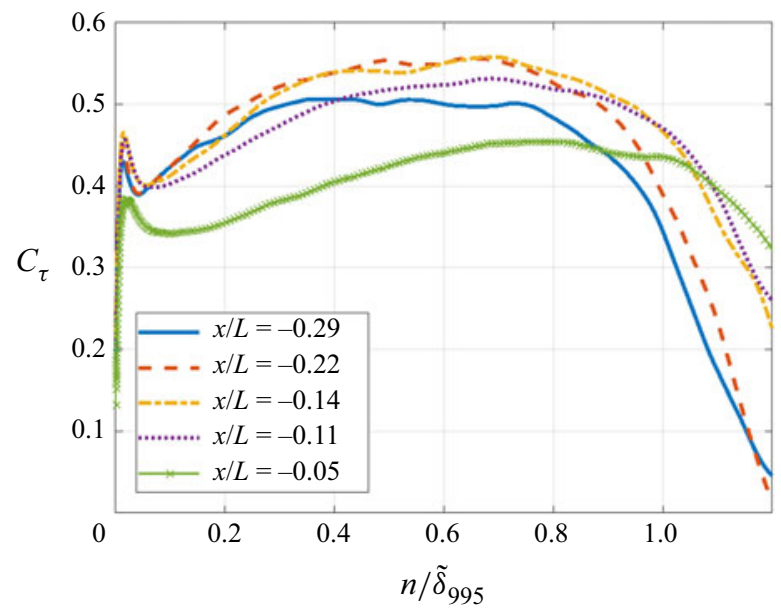

Figure 13. Correlation coefficient $C_{\tau}$ in the FPG region of the Gaussian bump.

(a)

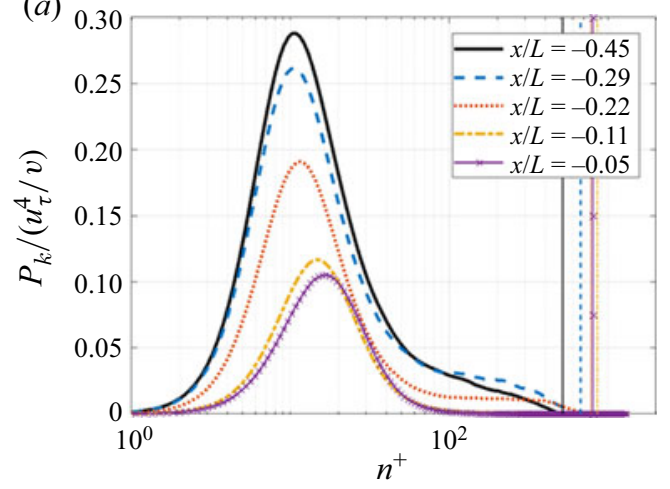

(b)

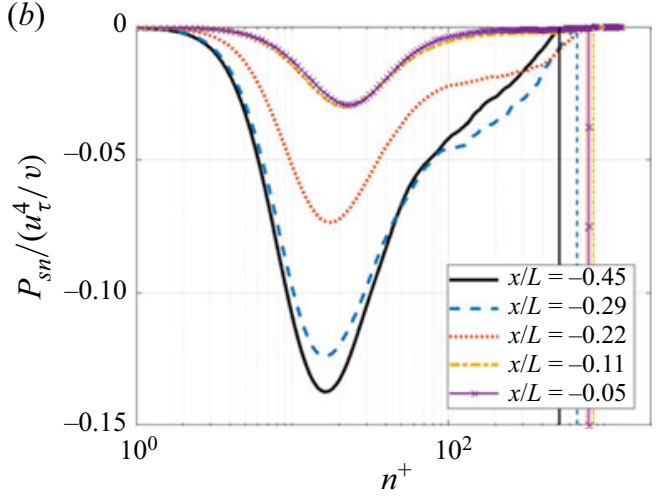

Figure 14. Production rate of the TKE ( $a$ ) and Reynolds shear stress (b) in the FPG region of the Gaussian bump non-dimensionalized by wall units. The vertical lines mark the boundary layer thickness $\tilde{\delta}_{995}$ for each streamwise location.

are defined, respectively, as

$$
P_{k}=-\overline{u_{i}^{\prime} u_{j}^{\prime}} \frac{\partial \bar{u}_{i}}{\partial x_{j}} \quad P_{s n}=-\left(\overline{u_{s}^{\prime} u_{k}^{\prime}} \frac{\partial \bar{u}_{n}}{\partial x_{k}}+\overline{u_{n}^{\prime} u_{k}^{\prime}} \frac{\partial \bar{u}_{s}}{\partial x_{k}}\right) .
$$

Note that Einstein notation is used to represent the double contraction and the indices $i, j$ and $k$ take the three dimensions of the curvilinear coordinate system $(s, n, z)$. Additionally, the profiles are non-dimensionalized by wall units, which allows a comparison of the production rates relative to the increase in wall shear due to the acceleration.

Immediately following the start of the FPG, a significant reduction in the magnitude of the inner layer peaks is observed. Given the destabilizing concave curvature of the streamlines upstream of $x / L=-0.14$, these changes are for the most part attributed to the strong pressure gradient. The peaks are also seen to move farther from the wall in $\mathrm{n}^{+}$units, indicating once again the thickening of the viscous sublayer. It is interesting to note that the peak non-dimensional TKE production for a ZPG flat plate boundary layer has a value of 0.25 (Jimenez et al. 2010), thus suggesting a reduction by a factor of 2.5 


\section{R. Balin and K.E. Jansen}

due to the strong acceleration and convex curvature. Farther from the wall $\left(n^{+} \gtrsim 100\right)$, both production rates in figure 14 decay rapidly and become negligible by $x / L=-0.11$, while still remaining positive in sign unlike the flow by Abe et al. (2012); Abe (2017) where negative production of TKE was found in the presence of convex curvature. Note that this decay is due to both the Reynolds stresses and the mean velocity gradient $\partial \bar{u}_{s} / \partial n$ diminishing significantly in the outer layer. Once again, since convex curvature is present only downstream of $x / L=-0.14$, this reduction is mainly attributed to the strong FPG, although curvature effects are contributing past this location. The strong acceleration, therefore, effectively turns off production in the outer layer, suggesting once again how this region behaves as a free shear layer with only a small dependence on the wall. Figure 14 therefore reveals once again the effects of a relaminarization process through a weakening of the near-wall turbulent production relative to the rising wall shear and acceleration. The profiles, however, do not become negligible in magnitude, giving further evidence of the fact that the relaminarization process does not complete and the flow over the bump is still partially turbulent.

\subsection{Bump peak and strong $A P G$}

As shown by the skin friction coefficient in figure 2 and the contours of instantaneous vorticity in figures 3 and 9, in the vicinity of the bump peak where the FPG is relaxed and pressure gradient changes from favourable to adverse there is a sudden enhancement of the near-wall vorticity which leads to a rise in the wall shear. This feature is of course related to the significant weakening of the near-wall turbulence caused by the strong upstream FPG and the partial relaminarization. It is, in fact, a partial retransition to fully turbulent flow, where the word 'partial' is used since the upstream flow did not reach the quasi-laminar state associated with the completion of the relaminarization process. In this section, the details of the flow and turbulence as they move through this segment of the bump are discussed.

Figure 15 shows slices of instantaneous vorticity magnitude normalized by the local time- and spanwise-averaged vorticity at the wall over the bump peak. The slices are taken at different heights within the boundary layer, in this case measured in wall units with $n^{+}$. Note that streamwise changes in mean $u_{\tau}$ are accounted for, thus the slice is at the same height above the wall in local wall units but not in physical units since $u_{\tau}$ is not constant. Moreover, black lines across the domain are used to mark the location of key events of the boundary layer flow. These are the peak strength of the FPG at $x / L=-0.11$, the bump peak and change in sign of the pressure gradient at $x / L=0.00$, the small local maximum in $C_{f}$ at $x / L=0.05$, and approximately halfway between the bump peak and incipient separation at $x / L=0.19$.

Starting with the slice closest to the wall at $n^{+}=5$, turbulent spots, which may be identified as clumps of small-scale turbulent structures of high intensity, are seen to form as early as upstream of the bump peak where the FPG is relaxed. These are intermittent with regions of very quiet and weak vorticity fluctuations, and appear to grow in size and intensity as they progress downstream into the strong APG. As noted by other studies on relaminarizing boundary layers (Blackwelder \& Kovasznay 1972; Narasimha \& Sreenivasan 1973; Sreenivasan 1982), these are clear signs of a retransition process taking place near the wall. As the FPG is relaxed, the stabilizing effect of the acceleration diminishes and instabilities are allowed to grow once again to produce a new fully turbulent internal layer. The presence of fluctuations of different scales in the incoming flow makes this process often very sudden and energetic, as is evident in figure 15. It is important to note that this phenomenon is a feature of the upstream strong FPG and 


\section{DNS of a boundary layer with strong pressure gradients}
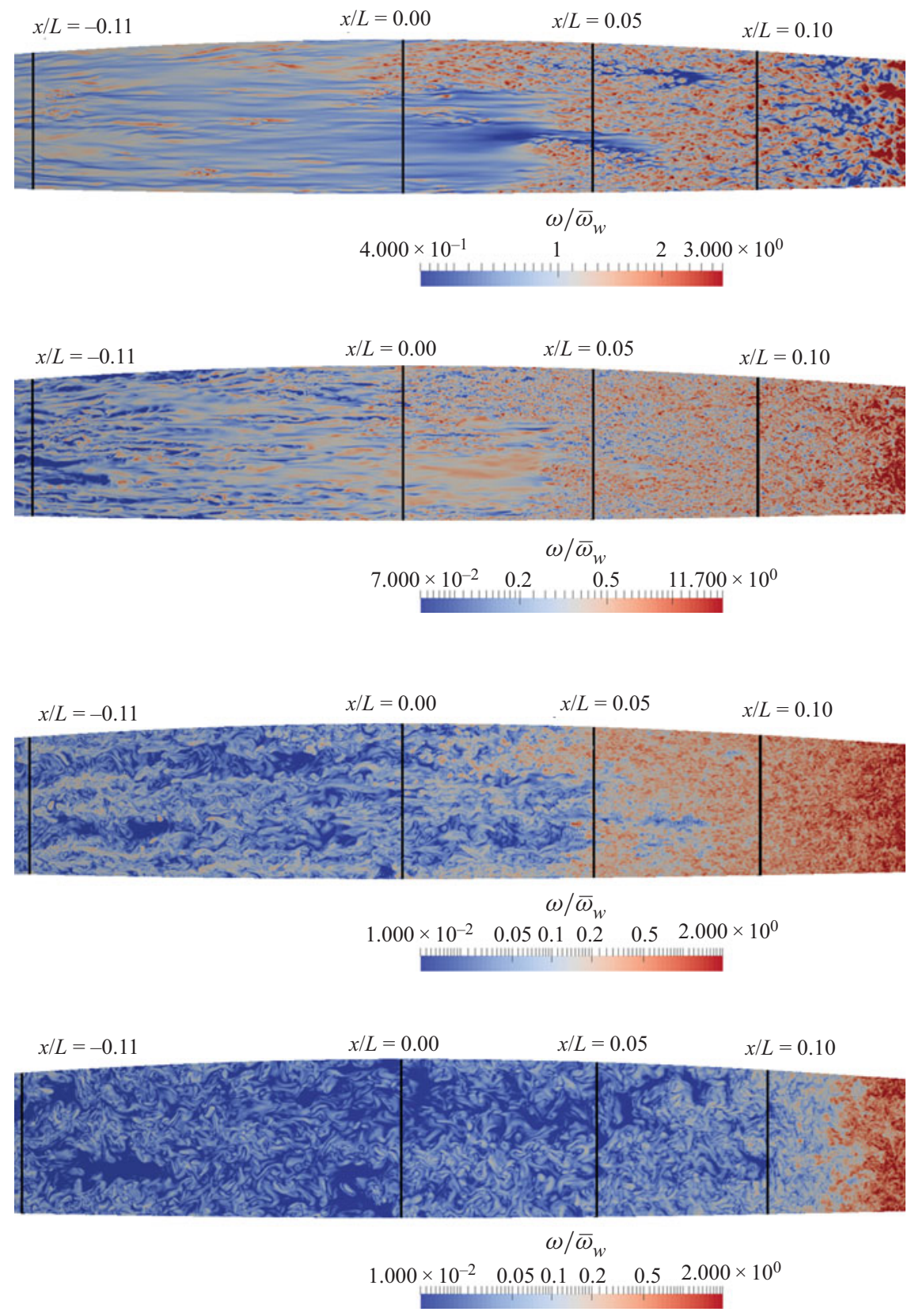

Figure 15. Instantaneous vorticity magnitude normalized by the local time- and spanwise-averaged wall vorticity at different locations within the boundary layer in the vicinity of the bump peak. From top to bottom, the heights above the wall of the slices are $n^{+}=5,30,100,300$. The black vertical lines mark the location of key events of the flow.

relaminarization process, and is not due solely to the APG. Previous studies exhibited this phenomenon even with the strong FPG relaxing into a ZPG region. Nevertheless, the destabilizing effects of the APG certainly aid in the onset of instability and growth of the turbulent spots, accelerating the formation of fully turbulent flow. At $x / L=0.05$, just four 


\section{R. Balin and K.E. Jansen}

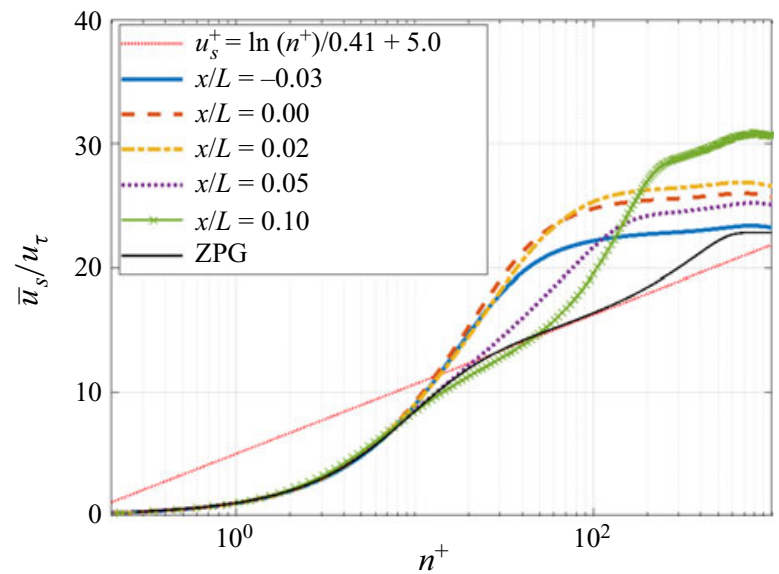

Figure 16. Mean streamwise velocity profiles in the APG region normalized by wall units. The ZPG profiles are taken from the DNS of Jimenez et al. (2010) at $\operatorname{Re}_{\theta}=1551$.

local boundary layer thicknesses downstream of the bump peak, the boundary layer at this height above the wall is effectively fully turbulent. The scales at this location are small and fairly isotropic in shape, but grow in size and stretch in the streamwise direction by $x / L=0.10$. The structure of the canonical boundary layer (see figure 9 at the start of the FPG) is not recovered, however, due to both upstream history and the strong APG.

The other slices in figure 15 show the height of the turbulent spots and their growth rate in the wall-normal direction. In their initial stages, the turbulent spots extend well past $n^{+}=30$ but are barely visible at $n^{+}=100$. Signs of the new internal layer only become visible farther downstream at this height. This indicates clearly that this phenomenon originates near the wall and propagates away from it as the internal layer grows into the boundary layer. The highest location above the wall, $n^{+}=300$, shows the flow in the outer wake of the boundary layer (see the velocity profiles in figure 16) as it flows from the FPG into the APG. In this region, no change in the size, structure and strength of the turbulent scales is visible from the contours of vorticity. The only change is observed downstream of $x / L=0.10$ when the internal layer finally reaches this height above the wall. This behaviour is consistent with the notion that the inner and outer layers of this flow are almost independent of each other, with the latter behaving similarly to a free shear layer subject to the strong convex curvature effects that are present around the peak of the bump. By contrast, the near-wall physics are dominated by the pressure gradient and their associated relaminarization and retransition that are not directly 'felt' in the outer layer.

The mean streamwise velocity profiles are presented in figure 16 for a number of locations around the bump peak and the initial part of the APG region. At $x / L=0.02$, which corresponds to the local minimum in the $C_{f}$ curve, the velocity still exhibits the effects of the upstream FPG, which are characterized by a steep velocity gradient in the thicker (in terms of $n^{+}$) viscous sublayer resembling a laminar flow profile and producing a large deviation above the standard logarithmic law. This is consistent with the contours in figure 15 since the flow is still heavily intermittent at this location. Farther downstream at $x / L=0.05$, the effects of the partial retransition become visible. The velocity gradient in the standard logarithmic region is reduced along with the thickness of the viscous sublayer due to the sudden surge in shear stress discussed later in this section. At the last station plotted, the velocity approaches the standard logarithmic law even closer and appears to 


\section{DNS of a boundary layer with strong pressure gradients}

have two distinct regions. The first is found below $n^{+}=40$ and resembles the canonical turbulent boundary layer shape with a buffer layer and a semilinear section (although with a different slope and intercept from the standard values). This is caused by a new internal layer forming at the bump peak which has reached heights around $n^{+}=100$ at this streamwise location. The second region appears to be a very pronounced wake above the underlying internal layer which maintains the shape of the upstream FPG profiles. Note that due to the strength of the APG and the nearing of incipient separation at $x / L=0.19$, the velocity does not reach agreement with the standard logarithmic law before separation. This result is consistent with the vorticity contours at the wall in figure 3 which show that the standard wall streaks do not develop within the APG region.

The Reynolds stresses for the same locations are presented in figure 17. Starting with the TKE, at the end of the FPG and at the bump peak the profiles look similar to the ones in the upstream FPG, with a larger peak than the $x / L=-0.05$ location in figure 11 . This is consistent with the increase in the inner peak of this stress component due to the acceleration of the flow. There is also a further reduction in the TKE of the outer layer due to the continued FPG and convex curvature effects. Entering the initial part of the APG, a significant surge in the value of the peak is observed, almost doubling at $x / L=0.05$ relative to the bump peak and then decreasing again after this station. This inner peak is also increasing in thickness with downstream location. These are clear signs of the new internal layer produced by the partial retransition. The outer layer TKE stays almost constant throughout the APG, providing further evidence of the independence of these two sections of the boundary layer. As the underlying internal layer grows in thickness, the outer layer is simply displaced farther from the wall, resulting in a significant growth of the overall boundary layer as seen in figure 4 .

The turbulent shear stress follows similar trends to the TKE, with some differences mainly in the outer layer. The inner peak also rises in magnitude and thickens significantly due to the new internal layer. Note that it is this sudden and significant surge in the near-wall turbulent shear caused by the partial retransition process that produces the local maximum in the skin friction observed in figure 2 at the start of the APG. In fact, this feature is typically observed downstream of a relaminarization process (Narasimha \& Sreenivasan 1979; Sreenivasan 1982; Warnack \& Fernholz 1998; Matai \& Durbin 2019). In the outer layer, the shear is continuously reduced, eventually changing sign at $x / L=0.10$. Since the pressure gradient effects are now destabilizing, these are clearly convex curvature effects that are still present until $x / L=0.14$. Once again, the inner part of the boundary layer is responding to the pressure gradient, while the outer layer is responding to the mean streamline curvature.

All three normal Reynolds stresses generally behave as expected, increasing near the wall and staying fairly constant or slightly decreasing in the outer layer. They do react to retransition and the APG slightly differently, however. The streamwise fluctuations respond the quickest, with the largest peak in figure 17 actually occurring at $x / L=$ 0.02. The wall-normal and spanwise fluctuations respond later but more drastically (the wall-normal fluctuations increase by a factor of seven near the wall in the interval $-0.02 \leq x / L \leq 0.05$ ), with the largest peak in the figure observed at the farthest downstream location shown. Clear changes in the anisotropy are also evident near the wall. While during the strong FPG and at the bump peak the streamwise fluctuations were dominating significantly over the other two directions, resulting in long and stretched streamwise-oriented structures, the normal Reynolds stresses become more balanced and isotropic during the partial retransition, although the streamwise direction still dominates. These trends in the span- and time-averaged profiles are consistent with the vorticity contours in figure 15. 

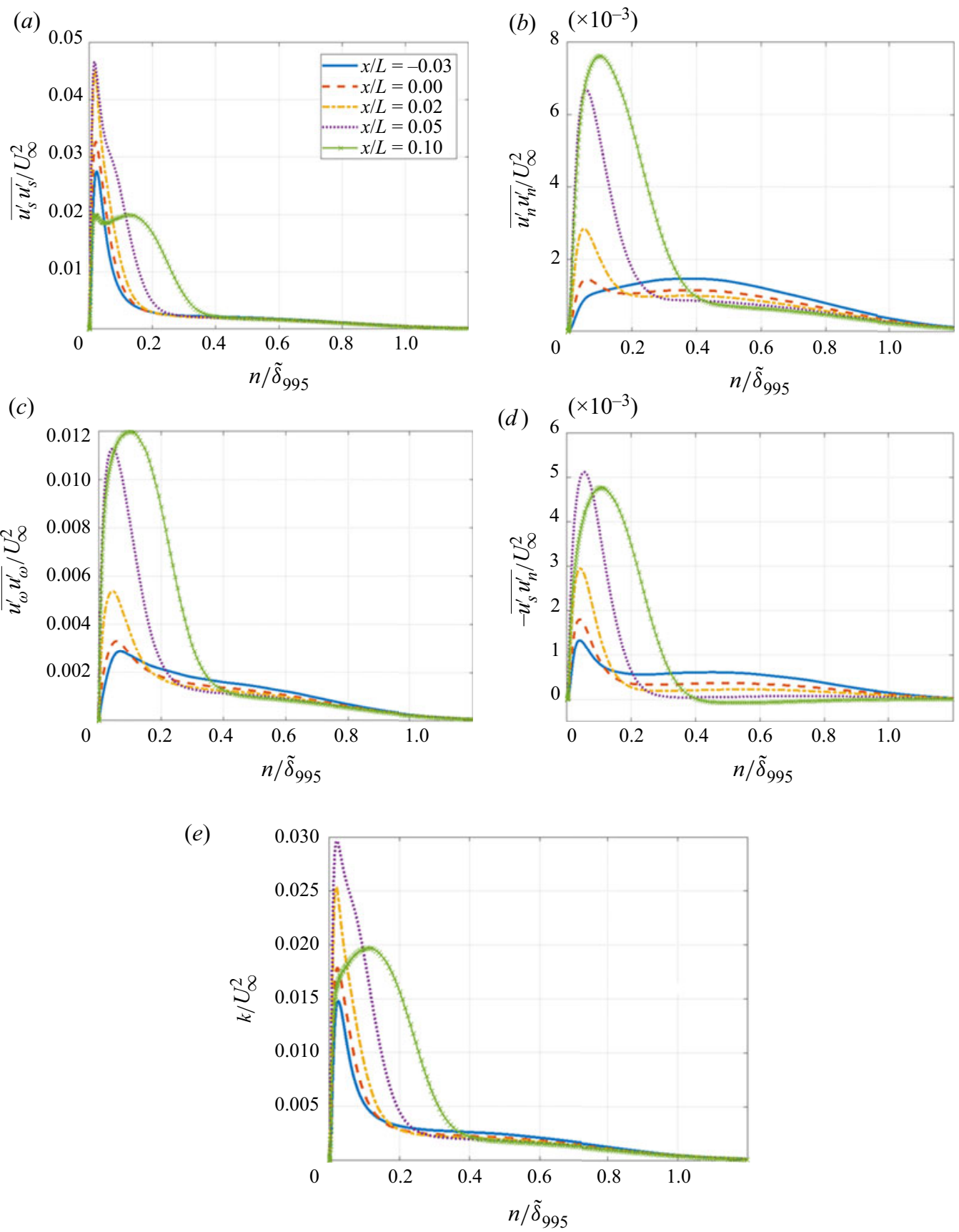

Figure 17. Mean Reynolds stresses and TKE in the APG region of the Gaussian bump normalized by $U_{\infty}$.

Figure 18 shows the correlation coefficient $C_{\tau}$ in the initial part of the strong APG. Relative to figure 13, the profiles at the end of the FPG and the bump peak show a slight reduction in correlation near the wall, but a more substantial reduction in the outer layer due to the convex streamline curvature. As the boundary layer moves through the APG, the correlation gradually increases near the wall and spreads away from it. This is another 


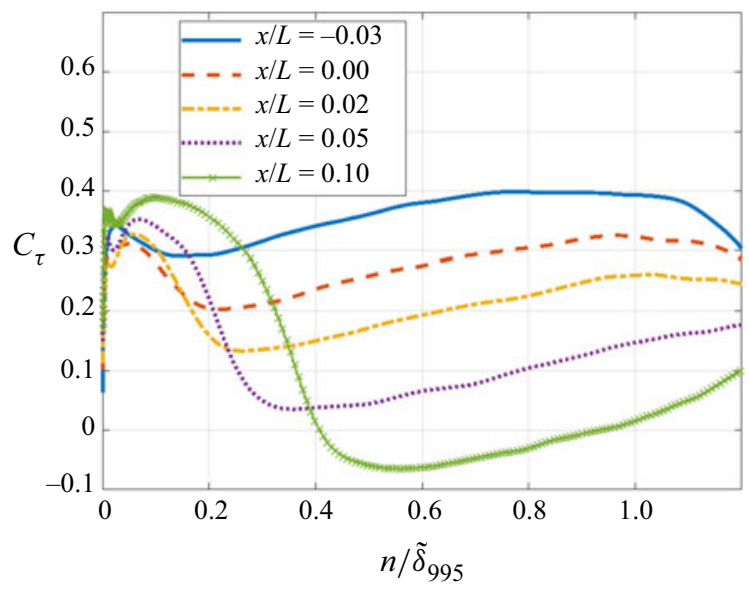

Figure 18. Correlation coefficient $C_{\tau}$ in the APG region of the Gaussian bump.

indication of the second internal layer. In the outer layer, $C_{\tau}$ keeps decreasing through the APG, reaching negative values by $x / L=0.1$. These profiles are in agreement with those reported in Narasimha \& Sreenivasan (1979) and So \& Mellor (1973), and show the strength of the curvature effects, as well as the region wherein they are active in the boundary layer. It is worth noting that a reduction in $C_{\tau}$ in the outer layer was also observed under strong APG and only mild curvature effects (Gungor et al. 2014; Abe 2019), although not to the same extent. Consequently, the pressure gradient is considered a contributing factor but not the dominant driver of the correlation in the outer layer of this flow.

The peak of the bump and the APG region exhibit a partial retransition process that develops a second internal layer with significantly increased turbulent intensity and wall shear. This newly energized flow is more resilient to the strong APG, and thus only exhibits incipient flow separation on the downstream side of the bump. Pressure gradient effects are focused in the near-wall region and are the main drivers of the skin friction coefficient, whereas the outer layer is fairly independent and shows the continued action of convex streamline curvature. It must be stated once again that the formation of this internal layer is directly dependent on the upstream FPG effects, and thus the two regions of the flow are highly connected. Indeed, a turbulence model hoping to accurately predict this bump flow must be capable of representing both of these complex physical phenomena.

\section{Conclusions}

Direct numerical simulation was performed of the turbulent boundary layer over a Gaussian-shaped bump. The smooth surface causes a series of alternating pressure gradients and mean streamline curvature effects which combine to form a multitude of complex flow physics with significant deviation from standard turbulence behaviour. The domain focused upon for this study is the portion of the boundary layer from the inflow to the point of incipient separation, with particular emphasis on the strong FPG.

Due to a strong acceleration, the boundary layer exhibits the stages of a relaminarization process. The standard logarithmic law breaks down soon after the start of the FPG and profiles of the streamwise velocity deviate significantly above it. The non-dimensional shear stress parameter, $\Delta_{\tau}$, is found to be predictive of the departure above the log law. Instantaneous contours of vorticity, however, indicate that the boundary layer is still fully 


\section{R. Balin and K.E. Jansen}

turbulent in this region of the flow, although the turbulent character and intensity are substantially altered by the FPG.

Under continued acceleration, the near-wall region gradually ceases to be fully turbulent and intermittent spots of quiet flow with low vorticity grow in size. This is considered to be the onset of relaminarization, which occurs in spite of the pressure and acceleration parameters never reaching the critical values of $\Delta_{p}=-0.025$ and $K=3 \times 10^{-6}$, respectively, suggested in previous studies. Relaminarization, however, does not complete and thus a quasi-laminar state is not achieved. In this region of the FPG, the Reynolds shear stress is reduced, developing a bimodal shape and the skin friction coefficient drops. When normalized by the local wall stress, the significant reduction of the TKE, the Reynolds stresses and their production rates clearly describes the weakening of the turbulence relative to the acceleration of the flow.

At the peak of the bump, as the pressure gradient changes to adverse, a partial retransition process is observed near the wall. The weakened flow from the upstream acceleration experiences a sudden enhancement in turbulent intensity, producing spots of large vorticity and a surge in TKE and Reynolds shear stress. The skin friction coefficient is also increased forming a local maximum. The newly energized boundary layer is more resilient to the strong deceleration on the downstream side, only resulting in incipient separation.

Two internal layers are also formed in the region of the flow focused upon, causing the inner and outer regions of the boundary layer to be largely independent of each other during the strong FPGs and APGs. The flow near the wall is dominated by the pressure gradients and is responsible for the skin friction coefficient, while farther from the wall the turbulence behaves similarly to a free shear layer subject to the continued effects of pressure gradients and streamline curvature. Curvature effects are found to be negligible in the near-wall region.

Reynolds averaged Navier-Stokes computations with the SA model of the same Gaussian bump flow resulted in a significant overprediction of the skin friction coefficient over most of the FPG and over the bump peak, as well as a much larger separation bubble due to the inability of the linear eddy viscosity closure to predict the correct pressure gradient effects. Similar results are observed with the shear stress stransport (SST) $k-\omega$ model in the context of wall-modelled LES (Balin et al. 2020). Future work is therefore focused on utilizing the DNS data for the improvement of RANS predictions of boundary layers under strong FPGs.

Acknowledgements. The authors thank Dr P.R. Spalart, Dr J.A. Evans and Dr M.K. Strelets for the helpful insight and communications regarding the problem set-up, the analysis of the flow and the synthetic turbulence generation method.

Funding. This work was supported by the National Science Foundation, Chemical, Bioengineering, Environmental and Transport Systems grant CBET-1710670 and by the National Aeronautics and Space Administration, Transformational Tools and Technologies grant 80NSSC18M0147, both to the University of Colorado Boulder. Computational resources were utilized at the NASA High-End Computing (HEC) Program through the NASA Advanced Supercomputing (NAS) Division at Ames Research Center and at the Argonne Leadership Computing Facility (ALCF), which is a DOE Office of Science User Facility supported under Contract DE-AC02-06CH11357.

Declaration of interests. The authors report no conflict of interest. 


\section{DNS of a boundary layer with strong pressure gradients}

\section{REFERENCES}

ABE, H. 2017 Reynolds-number dependence of wall-pressure fluctuations in a pressure-induced turbulent separation bubble. J. Fluid Mech. 833, 563-598.

ABE, H. 2019 Direct numerical simulation of a turbulent boundary layer with separation and reattachment over a range of Reynolds numbers. Fluid Dyn. Res. 51, 011409.

ABE, H. 2020 Direct numerical simulation of a non-equilibrium three-dimensional turbulent boundary layer over a flat plate. J. Fluid Mech. 902, A20.

Abe, H., Mizobuchi, Y., Matsuo, Y. \& Spalart, P.R. 2012 DNS and modeling of a turbulent boundary layer with separation and reattachment over a range of Reynolds numbers. In CTR Annu. Res. Briefs 2012, pp. 311-322. Center for Turbulence Research.

AntoniA, R.A. \& LuXTON, R.E. 1971 The response of a turbulent boundary layer to a step change in surface roughness. Part 1. Smooth to rough. J. Fluid Mech. 48, 721-761.

Antonia, R.A. \& LuXTON, R.E. 1972 The response of a turbulent boundary layer to a step change in surface roughness. Part 2. Rough-to-smooth. J. Fluid Mech. 53, 737-757.

Balin, R., JAnsen, K.E. \& Spalart, P.R. 2020 Wall-modeled LES of flow over a Gaussian bump with strong pressure gradients and separation. AIAA Paper 2020-3012.

Baskaran, V., Smits, A.J. \& Joubert, P.N. 1987 A turbulent flow over a curved hill. Part 1. Growth of an internal boundary layer. J. Fluid Mech. 182, 47-83.

Baskaran, V., Smits, A.J. \& Joubert, P.N. 1991 A turbulent flow over a curved hill. Part 2. Effects of streamline curvature and streamwise pressure gradient. J. Fluid Mech. 232, 377-402.

BlackWELDER, R.F. \& KovASZNAY, L.S.G. 1972 Large-scale motion of a turbulent boundary layer during relaminarization. J. Fluid Mech. 53, 61-83.

BRADSHAW, P. 1969 A note on reverse transition. J. Fluid Mech. 35, 387-390.

CAL, R.B. \& CASTILlo, L. 2008 Similarity analysis of favorable pressure gradient turbulent boundary layers with eventual quasilaminarization. Phys. Fluids 20, 105106.

CAVAR, D. \& MEYER, K.E. 2011 Investigation of turbulent boundary layer flow over 2D bump using highly resolved large eddy simulation. J. Fluids Engng 133, 111204.

Coleman, G.N., Rumsey, C.L. \& Spalart, P.R. 2018 Numerical study of turbulent separation bubbles with varying pressure gradient and Reynolds number. J. Fluid Mech. 847, 28-70.

Greenblatt, D., Paschal, K.B., Yao, C-S., Harris, J., Schaeffler, N.W. \& Washburn, A.E. 2006 Experimental investigation of separation control. Part 1: baseline and steady suction. AIAA J. 44, 2820-2830.

Gungor, A.G., Maciel, Y., Simens, M.P. \& SoriA, J. 2014 Analysis of a turbulent boundary layer subjected to a strong adverse pressure gradient. J. Phys.: Conf. Ser. 506, 012007.

Jansen, K.E., Whiting, C.H. \& Hulbert, G.M. 2000 Generalized- $\alpha$ method for integrating the filtered Navier-Stokes equations with a stabilized finite element method. Comput. Meth. Appl. Mech. Engng. 190, 305-319.

Jimenez, J., Hoyas, S., Simens, M.P. \& Mizuno, Y. 2010 Turbulent boundary layers and channels at moderate Reynolds numbers. J. Fluid Mech. 657, 335-360.

Kitsios, V., Atkinson, C., Sillero, J.A., Borrell, G., Gungor, A.G., Jimenez, J. \& Soria, J. 2016 Direct numerical simulation of a self-similar adverse pressure gradient turbulent boundary layer. Intl J. Heat Fluid Flow 61, 129-136.

MANHART, M. \& FRIEDRICH, R. 2002 DNS of a turbulent boundary layer with separation. Intl J. Heat Fluid Flow 23, 572-581.

MAtAi, R. \& DURbin, P. 2019 Large-eddy simulation of turbulent flow over a parametric set of bumps. J. Fluid Mech. 866, 503-525.

NA, Y. \& Moin, P. 1998 Direct numerical simulation of a separated turbulent boundary layer. J. Fluid Mech. 374, 379-405.

NARAsimha, R. \& SREenivasan, K.R. 1973 Relaminarization in highly accelerated turbulent boundary layers. J. Fluid Mech. 61, 417-447.

NARASimha, R. \& SREenivasan, K.R. 1979 Relaminarization of fluid flows. Adv. Appl. Mech. 19, $222-303$.

NARAyAnAN, M.A.B. \& RAMJEE, V. 1969 On the criteria for reverse transition in a two-dimensional boundary layer flow. J. Fluid Mech. 35, 225-241.

PAtel, V.C. 1965 Calibration of the Preston tube and limitations on its use in pressure gradients. J. Fluid Mech. 23, 185-208.

PATEL, V.C. \& HEAD, M.R. 1968 Reversion of turbulent to laminar flow. J. Fluid Mech. 34, 371-392.

SCHWARZ, A.C. \& PlesNiAK, M.W. 1996 Convex turbulent boundary layers with zero and favorable pressure gradients. J. Fluids Engng 118, 787-794. 


\section{R. Balin and K.E. Jansen}

Shur, M.L., Spalart, P.R., Strelets, M.K.. \& Travin, A.K. 2014 Synthetic turbulence generators for RANS-LES interfaces in zonal simulations of aerodynamic and aeroacoustic problems. Flow Turbul. Combust. 93, 63-92.

Shur, M.L., Strelets, M.K., Travin, A.K. \& Spalart, P.R. 2000 Turbulence modeling in rotating and curved channels: assessing the Spalart-Shur correction. AIAA J. 38, 784-792.

Skote, M. \& Henningson, D.S. 2002 Direct numerical simulation of a separated turbulent boundary layer. J. Fluid Mech. 471, 107-136.

SLOTNICK, J.P. 2019 Integrated CFD validation experiments for prediction of turbulent separated flows for subsonic transport aircraft. STO-MP-AVT-307.

So, R.M.C. \& Mellor, G.L. 1973 Experiment on convex curvature effects in turbulent boundary layers. J. Fluid Mech. 60, 43-62.

So, R.M.C. \& Mellor, G.L. 1975 Experiment on turbulent boundary layers on a concave wall. Aeronaut. Q. 26, 25-40.

SPALART, P.R. 1986 Numerical study of sink-flow boundary layers. J. Fluid Mech. 172, 307-328.

Spalart, P.R. \& Allmaras, S.R. 1994 A one-equation turbulence model for aerodynamic flows. Rech. Aerosp. 1, 5-21.

Spalart, P.R., Belyaev, K.V., Garbaruk, A.V., Shur, M.L., Strelets, M.K.. \& Travin, A.K. 2017 Large-eddy and direct numerical simulations of the Bachalo-Johnson flow with shock-induced separation. Flow Turbul. Combust. 99, 865-885.

Spalart, P.R. \& Coleman, G.N. 1997 Numerical study of a separation bubble with heat transfer. Eur. J. Mech. (B/Fluids) 16, 169-189.

SPALART, P.R. \& SHUR, M.L. 1997 On the sensitization of turbulence models to rotation and curvature. Aerosp. Sci. Technol. 5, 297-302.

SPALART, P.R. \& WATMUFF, J.H. 1993 Experimental and numerical study of a turbulent boundary layer with pressure gradients. J. Fluid Mech. 249, 337-371.

SREENIVASAN, K.R. 1982 Laminarescent, relaminarizing and retransitional flows. Acta Mech. 44, 1-48.

Trofimova, A.V., TejadA-Martinez, A.E. \& JAnsen, K.E. 2009 Direct numerical simulation of turbulent channel flows using a stabilized finite element method. Comput. Fluids 38, 924-938.

TSUJi, Y. \& MORIKAWA, Y. 1976 Turbulent boundary layer with pressure gradient alternating in sign. Aeronaut. Q. 27, 15-28.

Tsukahara, T., SeKi, Y. \& Kawamura, H. 2005 DNS of turbulent channel flow at very low Reynolds numbers. In Proceedings of the 4th International Symposium on Turbulence and Shear Flow Phenomena, pp. 935-940.

UzUN, A. \& MALIK, M.R. 2018 Large-eddy simulation of flow over a wall-mounted hump with separation and reattachment. AIAA J. 56, 715-730.

UZUn, A. \& MALiK, M.R. 2020 Simulation of a turbulent flow subjected to favorable and adverse pressure gradients. In AIAA AVIATION Forum. Virtual Event.

VOLINO, R.J. 2020 Non-equilibrium development in turbulent boundary layers with changing pressure gradients. J. Fluid Mech. 897, A2.

WARnACK, D. \& FERnhOlZ, H.H. 1998 The effects of a favorable pressure gradient and of the Reynolds number on an incompressible axisymmetric turbulent boundary layer. Part 2. The boundary layer with relaminarization. J. Fluid Mech. 359, 357-381.

Webster, D.R., DeGraAFF, D.B. \& EATON, J.K. 1996 Turbulence characteristics of a boundary layer over a two-dimensional bump. J. Fluid Mech. 320, 53-69.

Whiting, C.H. \& JANSEN, K.E. 1999 Stabilized finite element methods for fluid dynamics using a hierarchical basis. PhD thesis, Rensselaer Polytechnic Institute.

Williams, O., Samuell, M., Sarwas, E.S., Robbins, M. \& Ferrante, A. 2020 Experimental study of a CFD validation test case for turbulent separated flows. In AIAA Scitech 2020 Forum. Orlando.

Wright, J., BAlin, R., PAtterson, J.W., EvAns, J.A. \& JANSEN, K.E. 2020 Direct numerical simulation of a turbulent boundary layer on a flat plate using synthetic turbulence generation. arXiv:2010.04277.

Wu, X. \& SQUiRES, K.D. 1998 Numerical investigation of the turbulent boundary layer over a bump. J. Fluid Mech. 362, 229-271. 